Revue européenne des migrations internationales

vol. $25-n^{\circ} 1 \mid 2009$

Nouvelles migrations chinoises en Afrique et en Amérique latine

\title{
Ni invasion, ni exode
}

Regards statistiques sur les migrations d'Afrique subsaharienne

Neither Invasion nor Exodus. A statistical Overview of Migration from sub-

Saharan Africa

Ni invasión, ni éxodo. Estadisticos sobre las migraciones africanas de origen subsahariano

David Lessault et Cris Beauchemin

\section{(2) OpenEdition}

\section{Journals}

\section{Édition électronique}

URL : https://journals.openedition.org/remi/4889

DOI : $10.4000 /$ remi.4889

ISSN : $1777-5418$

Éditeur

Université de Poitiers

Édition imprimée

Date de publication : 1 juin 2009

Pagination : 163-194

ISBN : 978-2-911627-51-4

ISSN : 0765-0752

Référence électronique

David Lessault et Cris Beauchemin, « Ni invasion, ni exode », Revue européenne des migrations internationales [En ligne], vol. $25-n^{\circ} 1 \mid$ 2009, mis en ligne le 01 juin 2012, consulté le 23 novembre 2021. URL : http://journals.openedition.org/remi/4889; DOI : https://doi.org/10.4000/remi.4889 


\title{
$\mathrm{Ni}$ invasion, ni exode. Regards statistiques sur les migrations d'Afrique subsaharienne
}

\author{
David LESSAULT* et Cris BEAUCHEMIN**
}

La migration africaine d'origine subsaharienne occupe une place majeure dans les discours médiatiques et dans les dispositifs politiques européens dédiés à l'immigration. Cette focalisation sur les migrations africaines s'est affirmée à partir de 2005 . En septembre, les images des migrants prenant d'assaut la «forteresse Europe » en tentant de traverser les barrières qui séparent le Maroc des deux enclaves espagnoles de Ceuta et Melilla ont choqué les esprits. Deux mois plus tard, les chefs d'État et de gouvernement des pays de l'Union européenne, réunis à Hampton Court, ont conclu qu'il était urgent d'agir davantage pour maîtriser les migrations. La Commission a aussitôt répondu à cette attente en publiant une communication sur ses "priorités d'action en vue de relever les défis liés aux migrations ». Dans la foulée, s'inspirant de cette communication, le Conseil européen a adopté une «Approche globale sur la question des migrations » pour définir ses « priorités d'action centrées sur l'Afrique et la Méditerranée » (décembre 2005). Les conférences ministérielles Afrique-Europe sur les migrations de Rabat (2006), Tripoli (2006) et Paris (2008) découlent de ces priorités et témoignent de l'intérêt majeur que l'Europe porte à l'Afrique en tant que région émettrice de migrants. Parallèlement, plusieurs États membres de l'UE ont signé des accords de gestion des flux migratoires avec divers pays africains. Et le contrôle des frontières méridionales de l'Europe a été renforcé par les États membres eux-mêmes et par l'agence européenne Frontex de surveillance des frontières extérieures (Carling, 2007). Tout cet investissement politique, policier et diplomatique sur les migrations

* Chercheur post-doctoral, UMR CEPED, Université Paris Descartes, INED, IRD, 221 Boulevard Davout75020 Paris ; david.lessault@ined.fr

** Chargé de recherche, UMR CEPED, Université Paris Descartes, INED, IRD, 221 Boulevard Davout 75020 Paris ; cris.beauchemin@ined.fr

Nous remercions vivement Corinne Régnard, Xavier Thierry, Eléonora Castagnone, Pau Baizan et Amparo Gonzalez pour nous avoir permis d'accéder à leurs données et pour nous avoir apporté utilement les précisions nécessaires à notre compréhension. 
africaines — sans pareil pour les autres régions du monde — , associé aux images récurrentes de pirogues surchargées de migrants, suggère que déferle sur l'Europe une véritable « invasion africaine » (de Haas, 2007). Qu'en est-il réellement ? Rassemblant des données statistiques éparses, cet article donne la mesure des migrations subsahariennes en adoptant le double point de vue des pays de départ et d'arrivée, à l'échelle de la France mais aussi à l'échelle de l'Union Européenne ou de l'OCDE. En fin de compte, il met en évidence un paradoxe : les migrations subsahariennes à destination de la France ou, plus largement des pays du Nord, sont extrêmement visibles dans les discours politiques et médiatiques mais elles constituent un fait statistique minoritaire.

\section{L'IMMIGRATION SUBSAHARIENNE EN FRANCE}

Mobilisant les sources statistiques disponibles pour mesurer l'immigration en France (INSEE, AGDREF, OMI-STAT), nous examinons ici le poids relatif des Subsahariens dans la population étrangère et immigrée progressivement constituée. Au regard de la très faible représentation des migrants originaires d'Afrique Subsaharienne tant dans les flux récents (1994-2004) que dans les effectifs progressivement accumulés (1962-2005), une attention particulière est portée, dans l'analyse, à la contribution potentielle des migrations irrégulières. La prise en compte des migrations irrégulières, généralement tenues pour responsables d'une sous-estimation, est-elle de nature à bouleverser les ordres de grandeur ?

\section{2-2005 : Un stock en forte croissance mais toujours très marginal}

L'immigration subsaharienne (Encadré 1) en France se caractérise par la force de sa progression : depuis 1962, le nombre d'immigrés d'origine subsaharienne - personnes nées dans un pays d'Afrique subsaharienne et résidant en France au moment des recensements - a cru à un rythme moyen de $8,3 \%$ par an. C'est bien plus que le rythme de progression de l'ensemble de la population immigrée sur la même période (1,3\% par an). Les Subsahariens sont de fait, de toutes les origines, le groupe qui a le plus augmenté dans la période 1962-2004 (Tableau 1). Passant de 20000 à 570000 individus, la population subsaharienne a été multipliée par 27 en un peu plus de 40 ans.

\section{Encadré 1 : L'Afrique dont on parle}

L'Afrique subsaharienne, autrement appelée Afrique noire, regroupe l'ensemble des pays qui se trouvent au sud du Sahara. Les données présentées sont cependant tributaires des regroupements géographiques utilisés dans les différentes publications de données statistiques. En conséquence, la plupart des résultats présentés ici ne porte pas strictement sur l'Afrique subsaharienne mais sur l'Afrique hors Maghreb (Maroc, Algérie, Tunisie). L'assimilation de l'Afrique hors Maghreb à l'Afrique subsaharienne est géographiquement fausse puisque la première inclut l'Égypte et la Libye. Cependant, les effectifs libyens et égyptiens sont si faibles dans les flux et stocks d'immigrés en France que l'essentiel des effectifs présentés dans la catégorie Afrique hors Maghreb correspond, de fait, à l'Afrique subsaharienne. 
Tableau 1 : Évolution des stocks de la population immigrée en France par groupe d'origine (1962-2004)

\begin{tabular}{|l|r|r|r|r|r|r|r|}
\hline & \multicolumn{1}{c|}{$\mathbf{1 9 6 2}$} & $\mathbf{1 9 6 8}$ & \multicolumn{1}{c|}{$\mathbf{1 9 7 5}$} & $\mathbf{1 9 8 2}$ & $\mathbf{1 9 9 0}$ & \multicolumn{1}{c|}{$\mathbf{1 9 9 9}$} & $\mathbf{2 0 0 4}$ \\
\hline $\begin{array}{l}\text { Afrique hors } \\
\text { Maghreb }\end{array}$ & 20028 & 45934 & 93299 & 274952 & 391854 & 393289 & 570000 \\
\hline Maghreb & 406302 & 606996 & 995189 & 1065343 & 1103722 & 1298273 & 1500000 \\
\hline Europe & 2251827 & 2506729 & 2612373 & 2313221 & 2099639 & 1934144 & 1700000 \\
\hline Asie & 68670 & 82026 & 139948 & 322962 & 474918 & 549994 & 690200 \\
\hline $\begin{array}{l}\text { Amérique, } \\
\text { Océanie }\end{array}$ & 91560 & 36091 & 50536 & 64592 & 95816 & 130394 & 139800 \\
\hline Ensemble & 2861280 & 3281060 & 3887460 & 4037036 & 4165952 & 4306094 & 4930000 \\
\hline
\end{tabular}

Définition : Un immigré est une personne née à l'étranger, et qui ne possédait pas la nationalité française à sa naissance.

Source : INSEE.

- de 1962 à 1999 : Recensements de la population.

Tableau en ligne $:$ http://www.insee.fr/fr/ffc/accueil-ffc. asp?theme=2\&souspop=4

- pour 2004 : Enquêtes annuelles du recensement de 2004 et 2005. Résultats publiés in Insee

Première $n^{\circ} 1098$.

Cette progression spectaculaire s'explique en réalité par le très faible volume des Subsahariens en 1962. À l'époque, les Africains du sous-continent noir étaient les précurseurs d'une vague migratoire toute nouvelle. Les migrants originaires du sud de l'Europe puis du Maghreb les avaient devancés sur le territoire français. Ces derniers constituaient donc des effectifs relativement importants dans les années 1960 qui, du coup, ont cru à un rythme moins important dans les décennies suivantes (Garson, 1992).

Les taux de croissance inter-censitaire témoignent de la « jeunesse » de l'immigration africaine (Tableau 2). Dans le détail des périodes, la progression de la population subsaharienne n'est pas linéaire : très forte jusqu'aux années 1980, elle a notablement diminué à partir des années 1990. Ainsi les taux de croissance étaient-ils supérieurs à $10 \%$ par an jusqu'en 1982, avant de régresser jusqu'à 4,5\% entre 1982 et 1990, puis 2,7 \% entre 1990 et 2004. La période 1999-2004 semble cependant marquer une nouvelle accélération de l'immigration subsaharienne : le taux de croissance nul du début des années 1990 a laissé la place à un taux proche de $8 \%$ par an. Ce brutal retournement de tendance doit cependant être pris avec précaution. L'INSEE a en effet souligné dans ses propres publications que le recensement de 1999 a sous-estimé le nombre d'immigrés (Borrel, 2006). Si on assiste bel et bien à une reprise de l'immigration, elle est sans doute plus modérée que ce que suggère le chiffre ici présenté. On propose, en annexe 1, une ré-évaluation des effectifs comptabilisés en 1999. 
Tableau 2 : Taux de croissance annuels moyens de la population immigrée par période inter-censitaire (1962-2004)

\begin{tabular}{|l|r|r|r|r|r|r|r|r|}
\hline & $\mathbf{1 9 6 2 - 1 9 6 8}$ & $\mathbf{1 9 6 8 - 1 9 7 5}$ & $\mathbf{1 9 7 5 - 1 9 8 2}$ & $\mathbf{1 9 8 2}-1990$ & $\mathbf{1 9 9 0 - 1 9 9 9}$ & $\mathbf{1 9 9 9 - 2 0 0 4}$ & $\mathbf{1 9 9 0 - 2 0 0 4}$ & $\mathbf{1 9 6 2 - 2 0 0 4}$ \\
\hline $\begin{array}{l}\text { Afrique hors } \\
\text { Maghreb }\end{array}$ & $14,8 \%$ & $10,7 \%$ & $16,7 \%$ & $4,5 \%$ & $0,0 \%$ & $7,7 \%$ & $2,7 \%$ & $8,3 \%$ \\
\hline Maghreb & $6,9 \%$ & $7,3 \%$ & $1,0 \%$ & $0,4 \%$ & $1,8 \%$ & $2,9 \%$ & $2,2 \%$ & $3,2 \%$ \\
\hline Europe & $1,8 \%$ & $0,6 \%$ & $-1,7 \%$ & $-1,2 \%$ & $-0,9 \%$ & $-2,5 \%$ & $-1,5 \%$ & $-0,7 \%$ \\
\hline Asie & $3,0 \%$ & $7,9 \%$ & $12,7 \%$ & $4,9 \%$ & $1,6 \%$ & $4,6 \%$ & $2,7 \%$ & $5,6 \%$ \\
\hline $\begin{array}{l}\text { Amérique, } \\
\text { Océanie }\end{array}$ & $-14,4 \%$ & $4,9 \%$ & $3,6 \%$ & $5,1 \%$ & $3,5 \%$ & $1,4 \%$ & $2,7 \%$ & $1,0 \%$ \\
\hline Ensemble & $2,3 \%$ & $2,5 \%$ & $0,5 \%$ & $0,4 \%$ & $0,4 \%$ & $2,7 \%$ & $1,2 \%$ & $1,3 \%$ \\
\hline
\end{tabular}

Définition : Un immigré est une personne née à l'étranger, et qui ne possédait pas la nationalité française à sa naissance.

Source : INSEE.

- de 1962 à 1999 : Recensements de la population.

Tableau en ligne $:$ http://www.insee.fr/fr/ffc/accueil-ffc.asp?theme=2\&souspop=4

- pour 2004 : Enquêtes annuelles du recensement de 2004 et 2005. Résultats publiés in Insee Première $n^{\circ} 1098$.

En dépit de sa croissance, le groupe des Subsahariens a toujours été et demeure un groupe minoritaire dans l'ensemble de la population immigrée résidant en France. En 1962, on ne comptait que 20000 Subsahariens ; ils étaient cent fois moins nombreux que les immigrés d'Europe et vingt fois moins nombreux que les immigrés maghrébins. Ils représentaient alors moins de $1 \%$ de la population immigrée $(0,7 \%)$. Aujourd'hui, les Subsahariens restent trois fois moins nombreux que les Maghrébins ou les Européens et devancent seulement le groupe Amérique-Océanie. En 2004, avec environ 570000 individus, ils représentent un peu plus d'un dixième $(11,6 \%)$ de l'ensemble des immigrés (cf. Tableau 1 et Figure 1). Pour la plupart, ils sont originaires d'Afrique francophone (Encadré 2). Dans le palmarès des quinze pays d'origine les plus représentés dans la population immigrée, seuls le Sénégal et le Mali apparaissent, tenant respectivement les $13^{\text {ème }}$ et $15^{\text {ème }}$ places en 1999 comme en 2004 (Borrel, 2006).

Figure 1 : Évolution de la population immigrée en France par groupe d'origine (en millions d'individus)

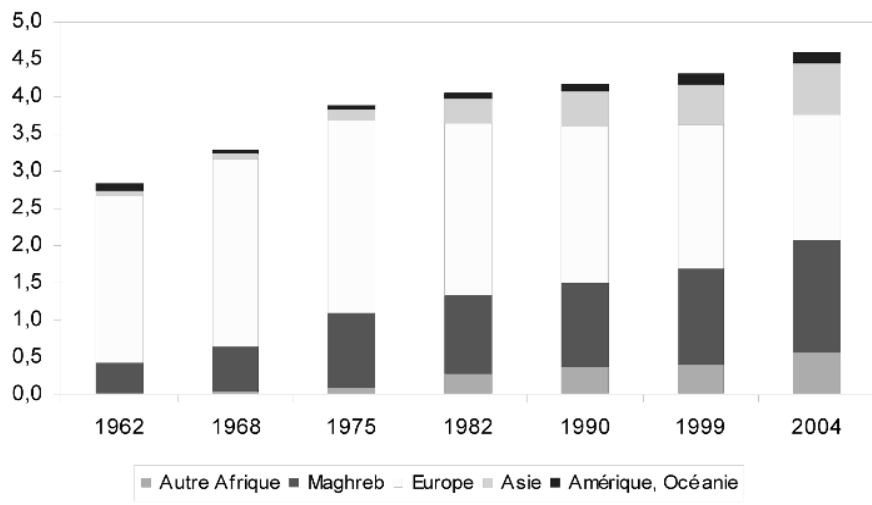




\section{Encadré 2 : Nationalités des immigrés subsahariens en 1999}

L'Afrique francophone est la région d'origine la plus représentée parmi les immigrés d'origine subsaharienne. Elle peut se décliner en trois bassins d'émigration d'inégale importance, en ordre décroissant: (1) l'Afrique de l'Ouest (Sénégal, Mali, Côte d'Ivoire, Cap Vert, Guinée) ; (2) l'Afrique centrale (Congo, Zaïre, Cameroun) ; (3) l'Afrique «insulaire » (Madagascar, Maurice, Comores). La plupart des nationalités les plus représentées correspondent aux territoires d'anciennes colonies françaises. De fait, le lien migratoire entre la métropole et ses anciennes colonies demeure fort : dans la période 1994-2004, trois entrées subsahariennes sur quatre trouvaient leur origine dans un territoire anciennement français (Tableau 3 et Figure 2).

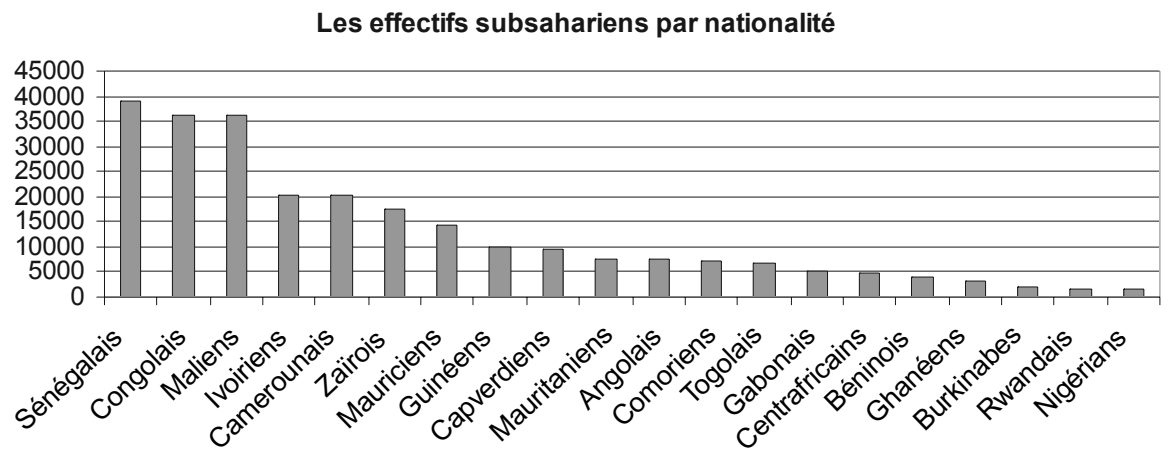

Source : INSEE, Mars 2002, CD ROM : Recensement 1999, La population immigrée étrangère en France.

Tous les immigrés ne sont pas étrangers : certains sont nés à l'étranger avec la nationalité française, d'autres l'ont acquise après immigration. Il n'existe pas - à notre connaissance - de tableau publié précisant les taux d'acquisition de la nationalité par groupe d'origine. Les publications de l'INSEE suggèrent néanmoins que ce taux est plus faible pour les Africains que pour les autres groupes. Il semble dépendre de l'ancienneté des courants migratoires. En 2004, ce taux était ainsi de $21 \%$ pour les Maliens contre $40 \%$ pour l'ensemble des immigrés (Borrel, 2006). Mais ce taux semble progresser rapidement : pour les individus originaires du Sénégal, il était d'environ $17 \%$ en 1990 et de $33 \%$ en 1999. Au total, en 1999, les 393289 immigrés d'origine subsaharienne comportaient une proportion non négligeable d'individus Français puisque le recensement dénombrait seulement 235569 personnes déclarant une nationalité subsaharienne (certains pouvant d'ailleurs détenir également la nationalité française). Les Subsahariens représentaient alors $12 \%$ de la population étrangère résidant en France métropolitaine (cf. Encadré 2 pour le détail de nationalités). De nouveau, ils forment une minorité. 


\section{4-2004 : les Subsahariens, un flux minoritaire}

De recensement en recensement, la population immigrée évolue en fonction des entrées et des sorties (décès ou émigration) de migrants. Nous ne disposons pas en France de données sur l'émigration en dehors du territoire français.

Une estimation globale est possible, mais elle ne rend pas compte du détail par nationalité ou grands groupes d'origine. L'évaluation des « sorties » est réalisée de manière indirecte. En réalité, on obtient une estimation du solde migratoire (EntréesSorties) par la soustraction du solde naturel au solde total. En 2004, l'INSEE a évalué le solde migratoire à 107500 personnes (INSEE, 2004), ce qui relativise de moitié le nombre des entrées $(210000)$. On ignore à quel point les Subsahariens sont représentés dans ce calcul. À l'évidence, il faudrait idéalement soustraire la part inconnue des Subsahariens ayant quitté le territoire afin de préciser le solde migratoire annuel pour le groupe des Subsahariens. Cette opération est impossible faute de données, mais elle relativiserait encore davantage le volume des flux observés. Nous devons donc nous contenter de l'analyse des flux d'entrées.

\section{Encadré 3 : Les sources de mesure de l'immigration en France}

Les statistiques administratives sur les flux d'immigration étrangère en France sont produites par divers organismes, chacun suivant sa propre logique institutionnelle, et aucun ne couvrant la totalité des entrées.

Les données de 1'ANAEM (Agence Nationale de l'Accueil des Étrangers et des Migrations, ex-OMI) comptabilisent toutes les personnes qui passent la visite médicale obligatoire parce qu'elles sont admises à séjourner en France pour 3 mois au moins. Jusqu'en 2004 ces données étaient communiquées dans l'annuaire des migrations internationales «OMI STAT» publié par l'OMI (Office des Migrations Internationales, 1994-2004). De 2004 à 2007 elles ont été publiées par la DPM (Direction de la Population et des Migrations).

Les tableaux publiés par la DPM, partiellement repris dans cet article (Tableau 4) sont construites à partir des données de l'ANAEM, de l'OFPRA et des Ministères de la Justice et de l'Intérieur. Ils portent sur l'immigration à caractère permanent des ressortissants de pays tiers. Elle se compose d'étrangers introduits, régularisés ou bénéficiaires d'un changement de statut qui reçoivent un premier titre de séjour d'une durée au moins égale à un an, à l'exception des étudiants (Régnard, 2006). Sur la mesure spécifique des régularisations, voir le tableau 4. Et pour plus de détails : Régnard (2006) et OMI (1994-2004)

Les données AGDREF (Application de Gestion Des Ressortissants Étrangers en France) comptabilisent tous les premiers titres de séjour délivrés en préfecture à des étrangers nés hors de France. Elles mesurent donc les entrées réelles de l'année ou de l'année précédente (pour ceux ayant obtenu un 
titre provisoire avant la délivrance du titre de séjour), les régularisations, les changements de statut (acquisition de la nationalité etc.) et les entrées de mineurs dans le cadre du regroupement familial. En revanche, elles ne comptabilisent ni les mineurs (hors regroupement familial) ni les saisonniers (qui ont seulement des contrats de travail). Pour plus de détails : Thierry (2004).

Depuis 1999, I'INED propose une totalisation du nombre d'entrées annuelles en France construite à partir des données du Ministère de l'Intérieur et de celles de l'Office des migrations internationales, en suivant les recommandations internationales. La statistique des premiers titres (AGDREF) est complétée par d'autres données, notamment pour appréhender les arrivées de mineurs qui ne sont pas détenteurs de titres de séjour. Les entrées de mineurs sont tirées des statistiques de l'ANAEM (Ex OMI) relatives aux mineurs ayant bénéficié d'une procédure de regroupement familial pour les ressortissants des pays tiers. "On obtient de la sorte l'évaluation la plus exhaustive possible de l'immigration en France (...) Cette série recense l'ensemble des étrangers admis à un séjour régulier en métropole pour une durée supérieure ou égale à un an, quels que soient leur nationalité, leur âge, leur situation familiale ou professionnelle » (Thierry X., 2004).

Pour plus de détails : http://www.ined.fr/fr/pop_chiffres/France/flux_immigration/ et Thierry (2000, 2001, 2004), Prioux (2003).

En effet, les données des administrations chargées de l'accueil des migrants et de la délivrance des titres de séjour nous renseignent assez précisément sur l'évolution et le détail des flux d'entrées. La Figure 2 illustre l'évolution des flux d'entrées en France entre 1994 et 2004. On y voit distinctement que le volume annuel de l'immigration a régulièrement cru pendant cette décennie. Toutes origines confondues, le nombre total d'entrées est passé de 120000 à 210000 (Tableau 3 et Figure 2). Tous les groupes d'origine, à l'exception des Européens, ont contribué à cette croissance des volumes de l'immigration. Les migrants d'Afrique subsaharienne sont cependant ceux, avec les originaires du Maghreb, dont la croissance relative a été la plus forte pendant la période : avec un taux de croissance moyen de 11,5\% par an (contre 5,8\% pour l'ensemble des entrées), le nombre des entrées des Subsahariens a presque triplé en dix ans. Ce résultat tend à corroborer la forte croissance du nombre d'immigrés subsahariens entre 1999 et 2004, même si cette croissance semble surestimée (cf. annexe 1).

En fait, la progression du nombre d'entrées entre 1994 et 2004 est d'autant plus marquée que le volume initial des immigrants subsahariens était très faible : en 1994, un migrant sur dix seulement était originaire d'Afrique noire (cf. Tableau 3 et Figure 2). Ils étaient à peine plus nombreux que les migrants provenant d'Amérique ou d'Océanie. C'est qu'au début des années 1990, l'immigration subsaharienne était presque tarie (voir la faible croissance du stock des immigrés dans cette période). Du 
Tableau 3 : Immigration annuelle en France selon le pays d'origine (1994-2004)

\begin{tabular}{|c|c|c|c|c|c|c|c|c|c|c|c|c|}
\hline & 1994 & 1995 & 1996 & 1997 & 1998 & 1999 & 2000 & 2001 & 2002 & 2003 & 2004 & \begin{tabular}{|l|}
$1994-$ \\
$2004 *$
\end{tabular} \\
\hline $\begin{array}{l}\text { Afrique hors } \\
\text { Maghreb }\end{array}$ & 11719 & 10179 & 10597 & 19329 & 28746 & 20453 & 23228 & 27227 & 31210 & 33618 & 34872 & $11,5 \%$ \\
\hline Maghreb & 23029 & 18431 & 18746 & 27286 & 36138 & 33553 & 40953 & 51526 & 63107 & 68040 & 65695 & $11,1 \%$ \\
\hline Europe & 61216 & 55680 & 54212 & 54233 & 59322 & 61194 & 61911 & 64010 & 67022 & 67797 & 64598 & $0,5 \%$ \\
\hline Asie & 13123 & 11177 & 11447 & 14972 & 19668 & 17759 & 21001 & 25234 & 29027 & 30346 & 29310 & $8,4 \%$ \\
\hline $\begin{array}{l}\text { Amérique, } \\
\text { Océanie }\end{array}$ & 9797 & 9216 & 9352 & 10256 & 11255 & 11499 & 12776 & 14083 & 14682 & 14958 & 14917 & $4,3 \%$ \\
\hline Total & 118884 & 104683 & 104354 & 126076 & 155129 & 144458 & 159869 & 182080 & 205048 & 214759 & 209392 & $5,8 \%$ \\
\hline \multicolumn{13}{|c|}{ Détail Afrique hors Maghreb } \\
\hline $\begin{array}{l}\text { Afrique ancien- } \\
\text { nement française* } \\
\text { dont: }\end{array}$ & 7577 & 7057 & 7550 & 11668 & 19083 & 14959 & 17993 & 21289 & 24297 & 26161 & 26947 & $13,5 \%$ \\
\hline Sénégal & 1249 & 1282 & 1257 & 2023 & 3175 & 2678 & 3422 & 3694 & 4163 & 3907 & 3920 & $12,1 \%$ \\
\hline Cameroun & 675 & 668 & 768 & 1239 & 1798 & 1499 & 2039 & 2672 & 3190 & 3724 & 4123 & $19,8 \%$ \\
\hline Côte d'Ivoire & 812 & 831 & 958 & 1402 & 2020 & 1688 & 2187 & 2648 & 3009 & 3594 & 3913 & $17,0 \%$ \\
\hline Mali & 441 & 416 & 491 & 1533 & 3962 & 2051 & 1856 & 2124 & 2244 & 2580 & 2533 & $19,1 \%$ \\
\hline Congo & 657 & 637 & 698 & 1028 & 1464 & 1327 & 1487 & 2109 & 2608 & 2621 & 2669 & $15,0 \%$ \\
\hline Madagascar & 901 & 814 & 825 & 973 & 1271 & 1285 & 1568 & 1839 & 1898 & 1983 & 1917 & $7,8 \%$ \\
\hline $\begin{array}{l}\text { Autres nationa- } \\
\text { lités d'Afrique } \\
\text { dont: } \\
\text { République démo- } \\
\text { cratique du Congo }\end{array}$ & 1293 & 3122 & 3047 & 2784 & 3620 & 1623 & 1369 & 5938 & 2426 & 2650 & 2935 & $8,5 \%$ \\
\hline Maurice & 678 & 651 & 540 & 1172 & 1406 & 846 & 799 & 821 & 886 & 768 & 740 & $0,9 \%$ \\
\hline Égypte & 385 & 302 & 390 & 649 & 841 & 648 & 732 & 789 & 798 & 917 & 848 & $8,2 \%$ \\
\hline
\end{tabular}

* Taux de croissance annuel moyen de la période 1994-2004.

Définitions :

- Immigrant : personne ayant obtenu un titre de séjour d'une durée minimale de un an.

- Afrique anciennement française : Bénin, Burkina Fasso, Cameroun, RCentrafricaine, Comores, Congo-Brazzaville, Djibouti, Gabon, Guinée, Côte d'Ivoire, Madagascar, Mali, Mauritanie, Niger, Sénégal.

Source : Xavier Thierry, INED, 2004, selon les données d'AGDREF et OMI-STAT.

Tableau en ligne : http://www.ined.fr/fr/pop_chiffres/France/flux_immigration/ 
Figure 2 : Volume des flux d'immigration annuel depuis 1994

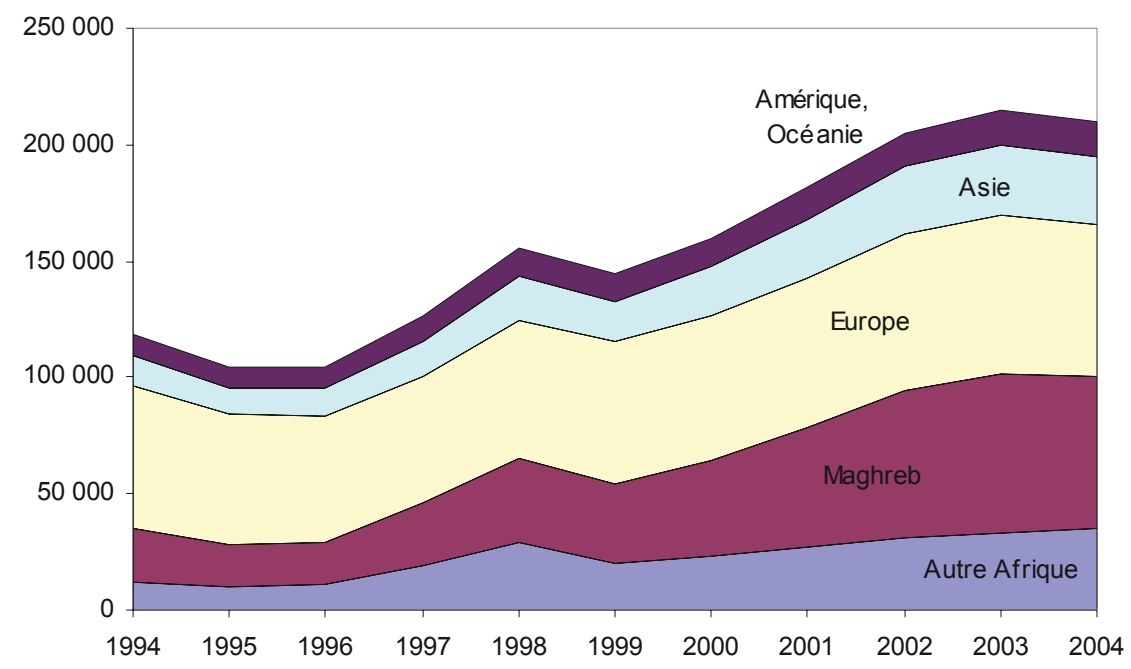

coup, en dépit de sa forte croissance dans les dix dernières années, l'immigration subsaharienne demeure un flux d'entrée très minoritaire en France. Sur l'ensemble de la période 1994-2004, les Africains (hors Maghreb) représentent seulement $15 \%$ des flux d'entrées dans le pays. À comparer, ils sont à peine plus nombreux que les Asiatiques et presque deux fois moins nombreux que les Maghrébins ou les Européens.

\section{Et les migrations irrégulières?}

Au constat que les migrations subsahariennes représentent un fait minoritaire, on pourrait objecter que les données de flux migratoires et d'effectifs subsahariens présentées ci-dessus n'incluent pas les migrations clandestines alors même que les Subsahariens sont souvent présumés alimenter largement ces flux. En réalité, les résultats présentés les incluent, au moins partiellement. D’une part, les données portant sur les effectifs, constituées à partir des recensements, peuvent inclure des personnes qui sont ou ont été en situation irrégulière ${ }^{1}$. D'autre part, les données de flux, puisqu'elles correspondent à la comptabilité des premiers titres de séjours octroyés aux migrants, incluent les individus qui ont été régularisés et qui, par définition, étaient antérieurement en situation irrégulière.

1 Malheureusement, aucune étude méthodologique - à notre connaissance - ne renseigne sur le degré de couverture de la population irrégulière par les opérations de recensement en France. 
Ainsi le pic de 1997-1998 qui se dessine clairement sur le graphique des volumes des flux d'immigration (cf. Figure 2) correspond-il à l'opération de régularisation entreprise par le gouvernement Jospin ${ }^{2}$. Cette hausse subite n'indique pas un surplus d'entrées en France mais plutôt un pic de délivrance de titres à des personnes entrées sur le territoire national depuis 6 ans en moyenne (Thierry, 2000). Ainsi, $41 \%$ des 185000 titres octroyés en 1997 et 1998 correspondraient à des régularisations (Tableau 4) ${ }^{3}$. Dans les années suivantes, les données de flux (cf. Figure 2 et Tableau 3) intègrent également les régularisations dites « au fil de l'eau » (par opposition aux régularisations liées à des opérations ponctuelles), instaurées par la loi RESEDA (1998) ${ }^{4}$. Au total, elles auraient concerné $13 \%$ des 950000 titres délivrés entre 1999 et $2006^{5}$.

Quelle place les migrants subsahariens prennent-ils dans ces dispositifs de régularisation? Tout indique qu'ils sont surreprésentés. Avec environ 30000 individus régularisés, ils représenteraient $40 \%$ de l'ensemble des migrants ayant bénéficié de l'opération de 1997-1998, alors que leur part dans l'ensemble des titres délivrés était de seulement $27 \%$ (cf. Tableau 4) ${ }^{6}$. Dans la période 1999-2006, 38500 personnes

2 Pour une brève présentation du cadre réglementaire de cette régularisation, voir Thierry (2000: 596).

3 La comptabilité du nombre des demandes et des régularisations effectives a fait l'objet de plusieurs estimations largement discutées par Thierry (2000 et 2001). Après ajustement des données d'AGDREF, il estime à 90000 le nombre de personnes ayant été régularisées en 1997 et 1998. On présente dans cet article des données inédites, issues de l'OMI, selon lesquelles il y aurait environ 76000 personnes ayant profité de l'opération de régularisation, certaines d'entre elles ayant pu être régularisées plus tardivement du fait des délais de traitement administratif qui se sont parfois étalés jusqu'en 2003. Les écarts d'effectifs de régularisés s'expliquent en grande partie par la vocation différente des administrations qui les ont produites (cf. Encadré 3) et par les différences de traitement des données : aucun ajustement des données de l'OMI n'a été fait, alors que les données d'AGDREF ont fait l'objet de plusieurs redressement explicités par Thierry $(2000,2001)$. On ne s'aventurera pas à proclamer qu'une source est plus juste que l'autre ; la réalité se trouve probablement entre les deux. On retiendra surtout que les statistiques sur les migrations, et a fortiori sur les régularisations, doivent être prises avec précaution.

4 Il s'agissait d'un système de régularisation permettant aux étrangers en situation irrégulière résidant en France depuis de nombreuses années d'obtenir un titre de séjour, en dehors des opérations exceptionnelles. Trois cas de régularisation étaient possibles : (1) étrangers justifiant de leur résidence habituelle en France depuis au moins dix années ; (2) mineurs ayant leur résidence habituelle en France depuis l'âge de 10 ans, ou 15 ans s'ils sont étudiants ; (3) étrangers dont les liens personnels et familiaux en France sont tels, que le refus d'autoriser leur séjour porterait, à leur droit, à leur vie privée et familiale, une atteinte disproportionnée au regard des motifs de refus (Régnard, 2006 : 31). Cette mesure de la loi RESEDA a été partiellement abrogée par la loi Hortefeux de 2007.

5 La vague de régularisation de 2006, entreprise par le Ministre de l'intérieur Sarkozy, n'entre pas dans ces résultats. Aucun indicateur ne permet en effet de distinguer dans les données de l'OMI les titres octroyés par régularisation des autres titres de séjour. L'exploitation ultérieure des données d'AGDREF devrait permettre d'estimer le nombre des bénéficiaires et d'identifier leurs caractéristiques.

6 D'après les données d'AGDREF non redressées, seulement 24144 Subsahariens auraient bénéficié d'une régularisation en 1997 et 1998 (communication personnelle de Xavier Thierry), contre 30570 d'après les données de l'OMI ici présentées (cf. Tableau 4). Ils représenteraient ainsi $27 \%$ de l'ensemble des personnes régularisés au lieu de $40 \%$. On voit bien, de nouveau, avec quelle prudence les données doivent être analysées. 
Tableau 4 : Régularisations en France (1997-2006)

\begin{tabular}{|c|c|c|c|c|c|c|c|c|}
\hline \multirow{3}{*}{$\begin{array}{l}\text { Région de } \\
\text { naissance des } \\
\text { personnes } \\
\text { régularisées } \\
\text { Afrique hors } \\
\text { Maghreb }\end{array}$} & \multicolumn{4}{|c|}{$\begin{array}{c}\text { Vague de régularisation de } \mathbf{1 9 9 7 - 1 9 9 8} \\
\text { Nombre de visites médicales } \\
\text { passées dans le cadre... }\end{array}$} & \multicolumn{4}{|c|}{$\begin{array}{c}\text { Régularisations au fil de l'eau. 1999-2006 } \\
\text { Nombre de visites médicales } \\
\text { passées dans le cadre... }\end{array}$} \\
\hline & \multicolumn{2}{|c|}{$\begin{array}{l}\text {... d'une procédure de } \\
\text { régularisation (1) }\end{array}$} & \multicolumn{2}{|c|}{$\begin{array}{l}\text {... d'une procédure de } \\
\text { délivrance d'un titre de } \\
\text { séjour (y compris pour } \\
\text { régularisation) (2) }\end{array}$} & \multicolumn{2}{|c|}{$\begin{array}{l}\text {... d'une procédure de } \\
\text { régularisation (3) }\end{array}$} & \multicolumn{2}{|c|}{$\begin{array}{l}\text {... d'une procédure de } \\
\text { délivrance d'un titre de } \\
\text { séjour (y compris pour } \\
\text { régularisation) (4) }\end{array}$} \\
\hline & 30572 & $40,0 \%$ & 49410 & $26,7 \%$ & 38500 & $31,5 \%$ & 215140 & $22,6 \%$ \\
\hline Maghreb & 25105 & $32,8 \%$ & 64329 & $34,8 \%$ & 38627 & $31,6 \%$ & 383934 & $40,3 \%$ \\
\hline Asie-Océanie & 14618 & $19,1 \%$ & 40396 & $21,8 \%$ & 19672 & $16,1 \%$ & 175255 & $18,4 \%$ \\
\hline Amérique & 2630 & $3,4 \%$ & 16572 & $9,0 \%$ & 15026 & $12,3 \%$ & 87056 & $9,1 \%$ \\
\hline Europe & 3548 & $4,7 \%$ & 14328 & $7,7 \%$ & 10332 & $8,5 \%$ & 91683 & $9,6 \%$ \\
\hline Total & 76473 & $100,0 \%$ & 185035 & $100,0 \%$ & 122157 & $100,0 \%$ & 953068 & $100,0 \%$ \\
\hline
\end{tabular}

Source : ANAEM (1994-2004) et Régnard (2006), données compilées par D. Lessault.

Les chiffres présentés dans ce tableau portent sur l'immigration à caractère «permanent » des ressortissants de pays tiers, mesurée sur la base des visites médicales, organisées par l'ANAEM, passées par les immigrés dans le cadre de la procédure de délivrance d'un titre de séjour (Régnard, 2006).

Dans les colonnes (2) et (4) figurent les étrangers introduits, régularisés ou bénéficiaires d'un changement de statut dans le cadre d'une procédure de délivrance d'un premier titre de séjour d'une durée au moins égale à un an, à l'exception des étudiants. Les principaux motifs de délivrance concernés sont les suivants : migrations de travail, réfugié, migration familiale, et visiteur. Parmi les motifs de délivrance des titres de séjour enregistrés par l'ANAEM, certains permettent d'identifier indirectement les titres octroyés par régularisation.

Pour la régularisation de 1997-98 (colonne 1), il s'agit des catégories « ré-examen » et « entrées des personnes au titre du regroupement familial/Familles régularisées, dont ré-examen », motifs qui apparaissent dans les fichiers jusqu'en 2003, les délais administratifs de traitement de certaines demandes ayant dépassé l'année 1998. Les chiffres présentés dans cette colonne couvrent donc la période 1997-2003.

Pour les régularisations « au fil de l'eau » (colonne 3), pratiquées depuis 1999 par application de la loi RESEDA, il s'agit des catégories «Résidents 10 ans », " Liens personnels et familiaux » et «Mineurs ayant leur résidence habituelle en France depuis l'âge de 10 ans ». Aucune catégorie, en revanche, ne permet d'isoler les effectifs des titres délivrés à l'occasion des vagues de régularisations engagées en 2006 par le ministre Sarkozy.

originaires d'Afrique noire auraient été régularisés « au fil de l'eau », soit $31 \%$ de l'ensemble des personnes ayant bénéficié de la nouvelle procédure RESEDA. Tous dispositifs confondus, sur les dix populations les plus représentées parmi les régularisés, la moitié correspond à des nationalités subsahariennes (Figure 3). Les Africains issus du sud du Sahara occupent ainsi, avec ceux qui proviennent du Maghreb, une place prépondérante dans les régularisations menées entre 1997 et 2006. 
Figure 3 : Classement des 10 premières nationalités régularisées

(\% comparés de la régularisation exceptionnelle 1997-98 et des régularisations au "fil de l'eau" 1999-2006)

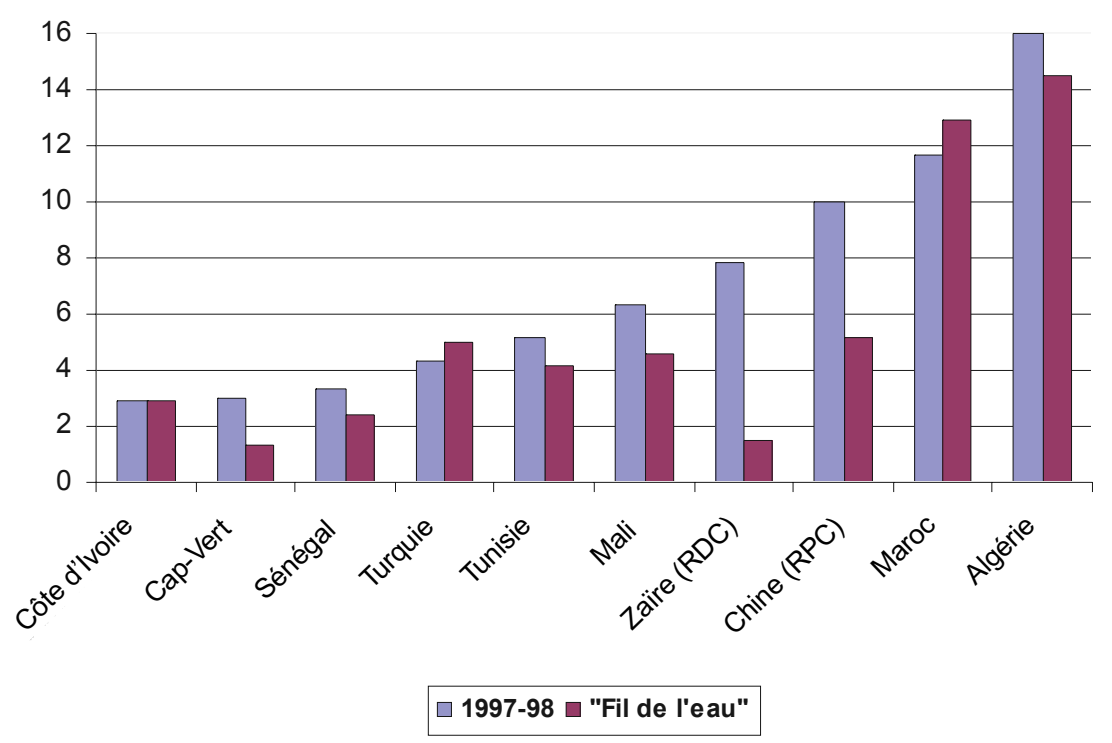

Comment expliquer cette surreprésentation des Subsahariens dans la population régularisée ? Au premier abord, elle n'est pas le produit d'un favoritisme administratif au moment du traitement des demandes car les demandeurs Subsahariens ont un taux de régularisation semblable à l'ensemble de la population des demandeurs ${ }^{7}$ (Thierry, 2001). En revanche, elle pourrait refléter, au moins en partie, le fait que les Africains (Subsahariens et Maghrébins) rencontrent plus d'obstacles que les autres populations pour entrer légalement en France. Les taux de refus relatifs à la délivrance des visas témoignent des restrictions qui s'appliquent à l'encontre des Africains : depuis la fin des années 1990, environ une demande sur cinq émanant de citoyens subsahariens est rejetée par les autorités consulaires, la proportion étant passée, entre 1998 et 2005, de 45 à $34 \%$ pour les Maghrébins, autre groupe ayant bénéficié largement des différentes formes de régularisation (Tableau 5). Par ailleurs, l'importance relative des demandeurs d'asile dans la population des régularisés indique que les régularisations viennent compenser - au moins en partie - les restrictions croissantes observées dans la délivrance du statut de réfugié. Par exemple, $48 \%$ des Congolais régularisés en 1997-1998 étaient des déboutés du droit d'asile (Thierry, 2000). Ces résultats conduisent à penser qu'il existe une relation entre régularisation et barrière à la migration légale : le nombre de régularisés serait inversement proportionnel au

7 Communication personnelle de Xavier Thierry détaillant les résultats présentés dans Thierry (2001). 
nombre de refus de visas, de titres de séjour, de statuts de réfugié. Pour étayer cette hypothèse, l'idéal serait de disposer de données, par pays d'origine, sur les taux de refus pour les années qui précèdent les différentes périodes de régularisation. Malheureusement, pour l'heure, ces données ne sont pas disponibles.

Tableau 5 : Délivrance des visas par zone géographique d'origine des demandeurs. France (1998-2005)

\begin{tabular}{|c|c|c|c|c|c|c|c|c|}
\hline \multirow[b]{2}{*}{ Régions } & \multicolumn{2}{|c|}{1998} & \multicolumn{2}{|c|}{2000} & \multicolumn{2}{|c|}{2004} & \multicolumn{2}{|c|}{2005} \\
\hline & Délivrés & Taux refus & Délivrés & Taux refus & Délivrés & Taux refus & Délivrés & Taux refus \\
\hline Maghreb & 250198 & $45 \%$ & 399144 & $40 \%$ & 403949 & $40 \%$ & 368144 & $34 \%$ \\
\hline Autre Afrique & 270343 & $18 \%$ & 306048 & $20 \%$ & 274275 & $22 \%$ & 269829 & $19 \%$ \\
\hline Asie-Océanie & 430536 & $7 \%$ & 363149 & $8 \%$ & 376384 & $8 \%$ & 391017 & $8 \%$ \\
\hline Europe de l'est & 478632 & $13 \%$ & 429160 & $16 \%$ & 421297 & $6 \%$ & 430957 & $6 \%$ \\
\hline Europe Occidentale & 186679 & $16 \%$ & 170170 & $19 \%$ & 165181 & $4 \%$ & 148890 & $5 \%$ \\
\hline $\begin{array}{l}\text { Proche et Moyen } \\
\text { Orient }\end{array}$ & 246538 & $7 \%$ & 279957 & $9 \%$ & 274921 & $9 \%$ & 306541 & $8 \%$ \\
\hline Amérique & 199482 & $6 \%$ & 166980 & $5 \%$ & 143453 & $8 \%$ & 138000 & $8 \%$ \\
\hline Total & 2062408 & $17 \%$ & 2114608 & $20 \%$ & 2059460 & $18 \%$ & 2053378 & $15 \%$ \\
\hline
\end{tabular}

Source : Haut Conseil à l'Intégration, 2008.

Quoi qu'il en soit, le constat de la surreprésentation des Subsahariens dans la population régularisée doit être nuancé. Les statistiques de régularisations permettent de battre en brèche deux idées reçues alimentées par les images médiatiques : selon la première, les irréguliers seraient essentiellement des noirs ; selon la seconde, ils arriveraient clandestinement aux portes de l'Europe au moyen de diverses embarcations. Commençons par la seconde. De manière générale, l'irrégularité du statut de séjour dans un pays à un moment donné n'implique pas que la personne est entrée illégalement dans ce pays ni qu'elle y a toujours vécu clandestinement. Dans la majorité des cas, les migrants entrent légalement, mais perdent leur droit de séjour à l'expiration de leur visa ou de leur premier titre de séjour. D'après une enquête, sur échantillon, réalisée au moment de la vague de régularisations de 1981-1982, seulement $5 \%$ des étrangers en situation irrégulière étaient entrés clandestinement sur le territoire (Marie, 1983). Plus récemment, les résultats des enquêtes Push-Pull (1997-1998) sur les Sénégalais en Espagne et les Ghanéens en Italie montrent aussi que les parcours migratoires conduisent très rarement à une entrée clandestine dans les pays de destination (Schoorl et al., 2000). Par ailleurs, les régularisés ont souvent connu des périodes de séjour légal avant même d'être régularisé. Ainsi, $41 \%$ des étrangers ayant demandé à être régularisés en 1997-1998 avaient déjà possédé des documents juridiques leur permettant de résider en France (récépissé, autorisation provisoire). C'était, par exemple, le cas de $61 \%$ des Congolais-Zaïrois, $41 \%$ des Maliens, $29 \%$ des Sénégalais (Thierry, 2001). 
Revenons à la première idée reçue. Malgré leur importance relative, les Africains subsahariens sont minoritaires : les populations issues des autres régions du monde totalisent $60 \%$ des titres octroyés pendant l'opération de 1997-1998 et $69 \%$ en 1999-2006 (cf. Tableau 4). Passant de 40 à $31 \%$, la part relative des Subsahariens est d'ailleurs déclinante entre les deux périodes. Cette tendance vaut pour tous les principaux groupes nationaux, à une exception près, celle de la Côte d'ivoire, pays qui précisément a connu une succession de troubles politiques depuis 1999 (cf. Figure 3) ${ }^{8}$. Quelle que soit la période considérée, l'Afrique subsaharienne ne figure pas dans le trio de tête des bénéficiaires des régularisations : l'Algérie, le Maroc et la Chine occupent ces places. Elle apparaît également fort peu dans les indicateurs de migrations irrégulières publiés dans le rapport annuel au Parlement sur l'immigration de 2007. Les résultats présentés, sous forme de palmarès par nationalité, ne permettent malheureusement pas d'isoler des résultats pour l'ensemble de l'Afrique sub-saharienne. Il en ressort néanmoins que les pays d'Afrique noire sont pratiquement absents des tableaux présentés, qu'il s'agisse des données sur les placements en zone d'attente, sur les refoulements à la frontière, sur les interpellations d'étrangers en situation irrégulière ou encore sur les mesures d'éloignement (Secrétariat Général du Comité Interministériel de Contrôle de l'Immigration, 2007).

En fin de compte, la prise en compte des situations d'irrégularité modifie-telle le constat fait que les Subsahariens occupent une place très minoritaire dans les stocks et les flux migratoires? De toute évidence, l'inclusion des irréguliers qui ont fait l'objet d'une régularisation ne change rien au constat initial puisqu'ils sont comptabilisés dans les données de flux présentées et, même probablement dans le recensement de 1999. Il reste les irréguliers qui n'ont pas été régularisés. Leur effectif est, en principe, inconnu. On peut toutefois estimer cette population en 1999 (Annexe 2). Quoi qu'il en soit, même en appliquant toutes les hypothèses qui tendent à majorer leur part relative dans la population, les Africains venus du Sud du Sahara demeurent une minorité dans la population immigrée (11,3\% au maximum), et encore plus dans la population résidant en France $(0,85 \%$, cf. Tableau 1 et 4$)$.

\section{LES MIGRATIONS SUBSAHARIENNES : MISES EN PERS- PECTIVE}

Minoritaires en France, les Subsahariens le sont aussi en Europe : en 1993, les personnes ayant la nationalité de l'un des 45 pays de l'Afrique subsaharienne représentaient seulement $6 \%$ de l'ensemble des étrangers de l'Europe des 15. La moitié d'entre eux était originaire d'Afrique de l'Ouest (Robin, 1996). Plus récemment, en 2000, les personnes originaires des pays situés au sud du Sahara représentaient seulement 3,8\% des migrants (personnes nées à l'étranger) installés dans les pays de l'OCDE (Tableau 6). En réalité, les Africains migrent peu en dehors de l'Afrique.

8 Préalablement, la part des Subsahariens avait beaucoup augmenté entre le début des années 1980 et la fin des années 1990 : à l'occasion de la vague de régularisation de 1981-1982, les Subsahariens représentaient seulement $15 \%$ des 130000 personnes régularisées (Garson, 1992). 


\section{En Afrique subsaharienne, une faible prévalence de la migration vers les pays développés}

Peu de données permettent de mesurer les migrations du point de vue des pays de départ, pour la simple raison que peu de pays sont équipés de dispositifs statistiques mesurant les sorties hors de leur territoire. Les pays africains n'échappent pas à cette généralité. Pour autant, deux sources sont mobilisables pour rendre compte de la prévalence de l'émigration internationale dans les pays subsahariens (Encadré 4) : (1) la compilation des recensements réalisée par l'OCDE et (2) le Réseau d'enquête sur les migrations et l'urbanisation en Afrique de l'ouest (REMUAO). Les résultats de l'OCDE et du REMUAO ne sont pas strictement comparables : les méthodes de mesure, les dates, les espaces géographiques diffèrent (Encadré 4). Pour autant, le fait frappant de ces deux sources de données est leur convergence vers un même résultat: la faible prévalence de l'émigration internationale en Afrique subsaharienne.

\section{Encadré 4 : Deux sources originales de mesure de l'émigration}

L'OCDE publie en ligne une base de données sur les stocks de migrants internationaux par nationalité dans les pays de l'OCDE. En 2003, l'OCDE a, en effet, lancé une collecte de données en collaboration avec les instituts nationaux de statistiques des pays membres afin d'obtenir des informations sur la population née à l'étranger. Cette base de données, dont la dernière mise à jour date de novembre 2005, permet de donner une vision d'ensemble de la migration vers chacun de ces pays au cours de la dernière décennie.

Pour plus de détails : Dumont et Lemaître (2005) et www.oecd.org/els/migration/DIOC

Le REMUAO (Réseau d'enquête sur les migrations et l'urbanisation en Afrique de l'ouest) est un ensemble d'enquêtes conduites en 1992 et 1993 dans 8 pays d'Afrique de l'ouest (Burkina Faso, Côte d'Ivoire, Guinée, Mali, Mauritanie, Niger, Nigeria et Sénégal) selon une méthodologie partout identique. Ce sont des enquêtes représentatives à l'échelle nationale qui, grâce à des questionnaires rétrospectifs, permettent de mesurer les flux de migration dans les 5 années qui précèdent chaque enquête. Un questionnaire ménage permettait d'identifier les personnes émigrées des ménages. Et des questionnaires biographiques individuels permettaient d'enregistrer tous les changements de résidence des personnes interrogées dans les 7 pays du réseau entre leur naissance et le moment de l'enquête.

Pour plus de détails : Bocquier et Traoré (2000)

D'après les données censitaires rassemblées par l'OCDE, le taux d'émigration des Subsahariens est de 0,9\% (Tableau 6). Autrement dit, moins d'une personne sur 100 née au sud du Sahara et âgée d'au moins 25 ans résidait dans un pays de l'OCDE en l'an 2000. Comparé aux autres régions du Sud, c'est peu : c'est à peine supérieur au taux d'émigration de l'Asie $(0,8 \%)$, c'est trois fois moins que celui de l'Afrique du 
nord $(2,9 \%)$ et plus de treize fois moins que celui de l'Amérique centrale $(11,9 \%)^{9}$. D'un point de vue plus qualitatif, les départs d'Afrique subsaharienne concernent une population plutôt plus instruite que dans les autres régions du monde. Le taux d'émigration est de $12,9 \%$ pour les plus qualifiés (personnes ayant au moins 13 années d'instruction), contre 5,5\% pour l'Asie ou 7,3\% pour l'Afrique du nord. Ainsi si la prévalence de la migration est trop faible pour affecter la dynamique démographique, ses effets économiques sont davantage en question du fait du «brain drain » qu'elle provoque (Docquier et Marfouk, 2006).

Tableau 6 : Émigration à destination des pays de l'OCDE.

Population âgée de 25 ans et plus (2000)

\begin{tabular}{|l|c|c|c|c|}
\hline & \multicolumn{2}{|c|}{$\begin{array}{c}\text { Part dans la population } \\
\text { des pays de l'OCDE (\%) }\end{array}$} & \multicolumn{2}{c|}{ Taux d'émigration (\%) } \\
\hline & $\begin{array}{c}\text { Ensemble des } \\
\text { migrants }\end{array}$ & $\begin{array}{c}\text { Migrants } \\
\text { qualifiés* }\end{array}$ & $\begin{array}{c}\text { Ensemble des } \\
\text { migrants }\end{array}$ & $\begin{array}{c}\text { Migrants } \\
\text { qualifiés }\end{array}$ \\
\hline Afrique & 7,6 & 6,8 & 1,5 & 10,4 \\
\hline Afrique subsaharienne & 3,8 & 4,7 & 0,9 & 12,9 \\
\hline Afrique du nord & 3,9 & 2,2 & 2,9 & 7,3 \\
\hline Amérique & 26,3 & 22,6 & 3,3 & 29,7 \\
\hline Amérique du Nord & 2,8 & 4,6 & 0,8 & 0,9 \\
\hline Amérique centrale & 13,7 & 6,6 & 11,9 & 16,9 \\
\hline Europe & 35,7 & 32,8 & 4,1 & 7,0 \\
\hline Asie & 25,5 & 34,5 & 0,8 & 5,5 \\
\hline Océanie & 1,4 & 1,8 & 4,3 & 6,8 \\
\hline
\end{tabular}

${ }^{1}$ Taux $=M /(M+N)$, où $M$ est le nombre de migrants (individus nés à l'étranger) enregistrés dans les recensements des pays de l'OCDE et $\mathrm{N}$ le nombre total de personnes résidant dans la région / le pays d'origine des migrants (indépendamment de leur nationalité).

2 Migrant qualifié : individu ayant reçu une instruction de 13 années au moins.

Source : Docquier et Marfouk (2006), Table 5.3.

9 Bien sûr, les taux d'émigration varient selon les régions et les pays. Selon une mesure alternative des taux d'émigration en 2000 à partir de la base de l'OCDE (le nombre de migrants n'est pas inclus dans le dénominateur, (cf. Tableau 6), Lucas (2006) estime que le taux était en moyenne de 4,5 pour 1000 pour l'ensemble de l'Afrique subsaharienne, les taux valant 4,2 pour l'Afrique de l'est, 5,1 en Afrique centrale, 7 en Afrique australe, et 4 en Afrique de l'ouest. Les pointes sont atteintes par les pays insulaires comme les Seychelles (96,2 pour mille), Maurice $(72,9)$, Sao Tome et Principe $(78,7)$, ou surtout le Cap Vert (191). À l'intérieur d'une sous région, les taux peuvent varier sensiblement passant par exemple de 0,5 au Niger à 21,5 pour mille en Guinée Bissau dans l'ensemble de l'Afrique de l'ouest continentale. Par ailleurs, Lucas montre que, en valeur absolue, l'Afrique de l'est est la première pourvoyeuse de migrants (environ un million d'individus comptabilisés dans les pays de l'OCDE en 2000), devant l'Afrique de l'ouest (900 000 migrants), l'Afrique centrale (470 000) et l'Afrique australe (350 000). Au total, presque 2,8 millions de Subsahariens vivaient ainsi dans l'un des pays de l'OCDE en 2000, soit 960000 de plus qu'en 1990. Malheureusement, les données présentées ne renseignent pas - à titre de comparaison - sur la progression de la population immigrante originaire des autres régions du monde. 
D’après les données du REMUAO (Tableau 7 ; Bocquier et Traoré, 1998), 22000 personnes ont quitté l'Afrique de l'Ouest pour rejoindre l'Europe en $1990^{10}$. Cela représente une personne sur 2500 , soit un taux d'émigration de 0,41 pour mille. Encore faut-il souligner que ces départs ont été compensés par 6600 migrations de l'Europe vers l'Afrique. Un départ sur trois de l'Afrique de l'Ouest vers l'Europe a ainsi été compensé par un retour. Cela confère finalement à l'Afrique de l'Ouest un taux net de migration de 0,29 pour mille, pour ce qui concerne ses échanges avec l'Europe. Avec des taux de migration aussi faibles, l'impact des migrations subsahariennes sur la croissance démographique des pays de départ et des pays d'accueil est négligeable. En 1990, l'apport migratoire de l'Afrique de l'Ouest à l'Europe représente 0,05 pour mille de la croissance démographique annuelle de l'UE, alors que celle-ci croît naturellement de 1,8 pour mille par an. Quant aux pays africains, la migration vers l'Europe a également une influence négligeable sur la dynamique démographique : elle représentait une baisse de 0,29 pour mille de la population en 1990, là où la croissance naturelle était de 28 pour mille, soit un rapport d'environ 1 à 100. En fin de compte, quel que soit le sens dans lequel on les interprète, les données du REMUAO montrent le caractère négligeable, du point de vue démographique, des

Tableau 7 : Volume des migrations internationales en Europe et en Afrique de l'ouest (1990)

\begin{tabular}{|l|c|c|}
\hline & Europe des 12 & Pays du REMUAO* \\
\hline $\begin{array}{l}\text { Migrations internationales } \\
\text { intra-continentales (a) }\end{array}$ & 600000 & 258000 \\
\hline $\begin{array}{l}\text { Migrations dans le sens } \\
\text { Afrique-Europe (b) }\end{array}$ & \multicolumn{2}{|c|}{22200} \\
\hline $\begin{array}{l}\text { Migrations dans le sens } \\
\text { Europe-Afrique (c) }\end{array}$ & \multicolumn{2}{|c|}{6600} \\
\hline Population totale (d) & 325000000 & 54000000 \\
\hline Taux pour 1000 (a)/(d) & 1,85 & 4,78 \\
\hline Taux pour 1000 (b)/(d) & 0,07 & 0,41 \\
\hline Taux pour 1000 (c/(d) & 0,02 & 0,12 \\
\hline Taux de migration nette (pour mille) & $+0,05$ & $-0,29$ \\
\hline Taux de croissance naturelle (pour 1000) & $+1,8$ & +28 \\
\hline
\end{tabular}

* Pays du REMUAO : Burkina Faso, Côte d’Ivoire, Guinée, Mali, Mauritanie, Niger et Sénégal. Nigeria exclu.

Sources : Tableau extrait de P. Bocquier (1998), «L'immigration ouest-africaine en Europe : une dimension politique sans rapport avec son importance démographique », La chronique du CEPED, juillet-Septembre $1998, \mathrm{n}^{\circ} 30$.

10 Il s'agit en réalité d'une moyenne annuelle établie sur la période 1988-1992. 
migrations entre l'Afrique de l'Ouest et l'Europe. Encore faut-il souligner que cette source tend à majorer l'évaluation de l'immigration en Europe par rapport aux sources officielles. En effet, dans la période 1988-1992, alors que le REMUAO a enregistré 111000 migrations des 7 pays du réseau vers l'Europe des 12, Eurostat n'en a enregistré que 23000 entrées en provenance des mêmes pays (Bocquier et Traoré, 1998). Les effectifs estimés à partir des enquêtes du REMUAO sont ainsi cinq fois supérieures aux enregistrements officiels des pays européens d'accueil ${ }^{11}$.

\section{La France et l'Europe ne sont pas les principales destinations des migrants subsahariens}

Si les migrations ont un impact aussi faible sur la croissance démographique de l'Afrique de l'Ouest ou de l'Europe des 12, c'est que les migrants subsahariens se tournent peu vers l'Europe. Car, avant toute chose, les migrations internationales des subsahariens sont des migrations intra-africaines (cf. Tableau 7). Les données du REMUAO montrent que les migrations vers l'Europe avaient, en 1990, un volume mineur (22 200) en comparaison du volume des flux intérieurs à l'Afrique (258 000). En fait, la mobilité intra-africaine (entre pays du REMUAO) s'avère extrêmement élevée : en une année, pour 1000 individus, 5 personnes ont changé de pays en Afrique de l'Ouest (indice d'intensité migratoire de 4,78 pour mille) quand moins de 2 personnes changeaient de pays au sein de l'UE (indice d'intensité migratoire de 1,85 pour mille).

La prédominance des flux intracontinentaux caractérise aussi les mouvements d'une catégorie particulière de migrants : les réfugiés. La répartition des pays de destination des réfugiés subsahariens montre clairement que les États voisins jouent un rôle prépondérant dans l'accueil des populations réfugiées (Tableau 8). Ainsi, en 1999, 9 réfugiés sur 10 originaires de la République Démocratique du Congo étaient restés en Afrique ; $2 \%$ seulement des 252400 réfugiés congolais se trouvaient alors en France. Il en était de même pour $96 \%$ des réfugiés libériens, $93 \%$ des Tchadiens et $88 \%$ des Rwandais. Ces proportions révèlent la portée géographique très limitée des déplacements de population liés aux conflits dans cette partie du monde. Les États européens, et la France en particulier, sont des acteurs très marginaux de l'accueil des populations réfugiées issues d'Afrique subsaharienne ${ }^{12}$.

11 Les résultats du REMUAO sont relativement anciens. Pour l'heure, aucune source ne permet de les actualiser. Pour autant, on peut faire l'hypothèse que la crise politique et militaire traversée par la Côte d'Ivoire depuis 1999 a substantiellement modifié les logiques migratoires régionales. Elle a sans doute provoqué (1) un mouvement de retour au pays d'une partie des Burkinabè et des Maliens qui étaient implantés dans le Sud de la Côte d'Ivoire, (2) une réduction notable des migrations à partir des pays voisins vers la Côte d'Ivoire, et (3) l'accélération de l'émigration ivoirienne hors du continent, comme en témoigne d'ailleurs les résultats présentés plus haut sur l'immigration en France.

12 Dans le contexte français, la population immigrée d'Afrique subsaharienne se caractérise néanmoins par l'importance des personnes ayant reçu un titre de séjour "réfugié ou apatride ». En 2004, sur 34025 Subsahariens ayant reçu un titre, 11 \% l'ont obtenu au nom 
Tableau 8 : Pays d'accueil des 4 premiers groupes d'origine des réfugiés d'Afrique subsaharienne en 1999

\begin{tabular}{|l|l|r|r|}
\hline Pays d'origine & \multicolumn{1}{|c|}{ Pays d'accueil } & Effectif & \% \\
\hline \multirow{5}{*}{ Congo (RDC) } & Pays d'Afrique subsaharienne & 235500 & 93,3 \\
& France & 5200 & 2,1 \\
& Canada & 2300 & 0,9 \\
& Autres pays & 9400 & 3,7 \\
\cline { 2 - 4 } & Total & 252400 & 100 \\
\hline \multirow{3}{*}{ Libéria } & Pays d'Afrique subsaharienne & 281700 & 97,7 \\
& États-Unis & 2500 & 0,9 \\
& Autres pays & 4200 & 1,5 \\
\cline { 2 - 4 } & Total & 288400 & 100 \\
\hline \multirow{3}{*}{ Tchad } & Pays d'Afrique subsaharienne & 55700 & 96,2 \\
& Autres pays & 2200 & 3,8 \\
\cline { 2 - 4 } & Total & 57900 & 100 \\
\hline \multirow{2}{*}{ Rwanda } & Pays d'Afrique subsaharienne & 74700 & 88,1 \\
& Belgique & 2100 & 2,5 \\
& Autres pays & 8000 & 9,4 \\
\cline { 2 - 4 } & Total & 84800 & 100 \\
\hline
\end{tabular}

Source : UNHCR (2000), «Les réfugiés dans le monde. 50 ans d'ans d'actions humanitaires », Annexes statistiques, pp. 301-325.

Plus généralement, les données censitaires compilées par la Banque mondiale et l'Université du Sussex confirment l'orientation essentiellement intra-africaine de l'ensemble des flux subsahariens, réfugiés ou non : en l'an 2000, sur 100 personnes nées dans un pays d'Afrique de l'Ouest où elles ne résidaient plus, 61 vivaient dans un autre pays de la sous-région (Afrique de l'Ouest), 8 vivaient en Afrique centrale et 1 en Afrique du nord ; restaient - hors du continent africain - 15 personnes en Europe, 6 en Amérique du Nord et 9 personnes dispersées dans divers pays (de Haas, 2007 : 30). Cependant, ces moyennes sous-régionales masquent le fait que certains pays sont majoritairement tournés vers des destinations extracontinentales : en 2000, le Cap Vert, la Côte d'Ivoire, le Gabon, la Gambie et la Sierra Leone comptent plus de la moitié de leurs expatriés aux États-Unis et en Europe (de Haas, $2007: 30)^{13}$.

de leur statut de réfugié ou d'apatride. La proportion moyenne, toutes origines confondues, était seulement de $5 \%$. Cette donnée reflète le fait que l'Afrique est davantage travaillée par des conflits que les autres régions du monde (source : Thierry selon les données d'AGDREF et OMISTAT, 2004). Tableau en ligne : http://www.ined.fr/fr/pop_chiffres/France/flux_immigration.

Cette surreprésentation statistique des Subsahariens reflète l'état géopolitique d'un continent travaillé par de multiples conflits (Cambrézy, 2001).

13 Pour une présentation détaillée des différents systèmes migratoires africains, voir : Spaan et van Moppes, 2006. 
Pour ceux qui quittent l'Afrique, l'Europe apparaît comme la principale destination : en 2000, les pays européens regroupent en effet presque les deux tiers $(63 \%)$ des Africains résidant dans les pays de $1^{\prime} \mathrm{OCDE}^{14}$. L'Amérique du Nord accueille un peu moins du tiers restant $(31 \%)$, une proportion non négligeable qui montre que les destinations des Subsahariens ne sont pas exclusivement européennes. Le classement des pays (plutôt que des grandes régions, (Figure 4) montre d'ailleurs que les États-Unis sont la première destination des Africains nés au sud du Sahara (24\% des Subsahariens de l'OCDE), avant la Grande Bretagne (21\%) et la France $(15 \%)$.

Figure 4 : Principales destinations des Subsahariens dans les pays de l'OCDE (2004)

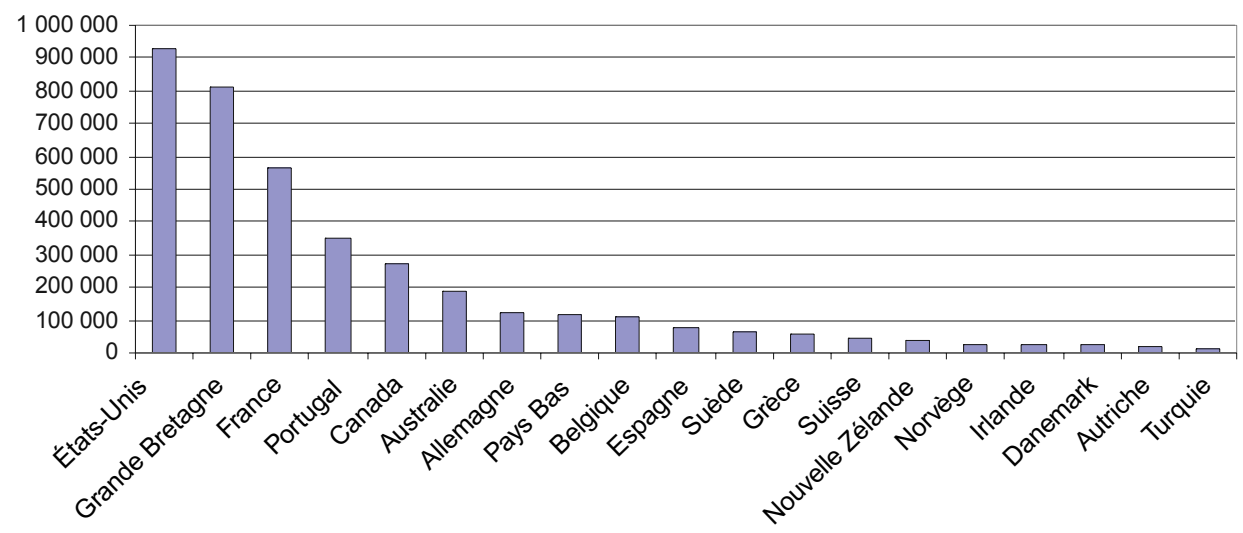

Dans le classement des principales destinations européennes, la France est en recul : aujourd'hui seconde (cf. Figure 4), elle était la première destination en 1993, devant l'Allemagne et la Grande-Bretagne (Robin, 1996). Depuis, de nouvelles destinations ont émergé, notamment au Sud de l'Europe. Cette évolution témoigne de la diversification des trajectoires des migrants subsahariens, qui tendent à délaisser les anciennes destinations privilégiées que constituaient les ex-métropoles coloniales. C'est ce dont témoignent les cas sénégalais ou ghanéens (Figure 5) : ces populations se dispersent en Europe au lieu de se concentrer presque exclusivement dans les territoires des anciennes puissances coloniales, comme c'était le cas auparavant ${ }^{15}$. Cette nouvelle distribution des migrants procède sans doute d'un effet des politiques migratoires restrictives des anciens pays de destination. Elle est aussi certainement liée à des différentiels d'opportunités économiques pour les migrants.

14 Résultats calculés par les auteurs à partir de la base de données de l'OCDE (Encadré 4).

15 Utilisant des informations recueillis dans quelques régions des pays de départ sur les destinations des migrants récents, les travaux du projet Push-pull conduisent aux mêmes conclusions : rôle minoritaire de l'Europe dans l'univers des destinations ghanéennes et sénégalaises et diversification des destinations au sein de l'Europe (Schoorl et al., 2000). 
Figure 5 : Principales destinations des Sénégalais et des Ghanéens en Europe en 2004 (Eurostat)

\section{Sénégalais}

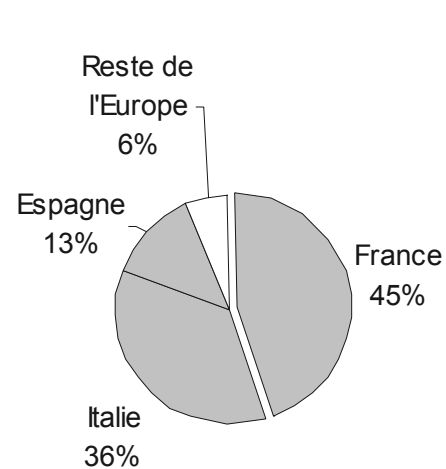

\section{Ghanéens}

Reste de

l'Europe

$9 \%$

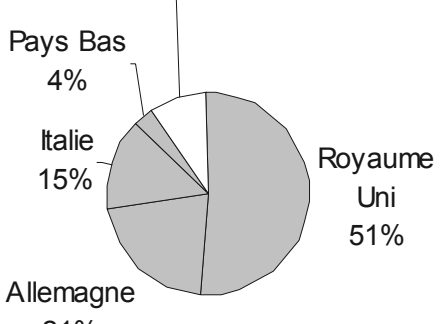

$21 \%$

\section{Les nouveaux pays de destination en Europe : des havres pour clandestins africains?}

La redistribution des flux subsahariens a fait émerger de nouveaux pays de destination à l'intérieur de l'Europe. L'Espagne et l'Italie, et dans une moindre mesure le Portugal et la Grèce, sont aujourd'hui des destinations prisées des migrants qui partent d'Afrique noire. C'est d'ailleurs au sud de l'Europe, au plus proche de l'Afrique, que l'agence Frontex renforce les frontières de l'Union européenne. C'est encore là qu'ont eu lieu les plus importantes vagues de régularisation au tournant du XX ${ }^{\mathrm{e}}$ siècle (Italie : 1998 et 2002 ; Espagne : 2000-2001 et 2005).

Tableau 9 : Espagne et Italie (2006). Répartition de la population immigrante (née à l'étranger)

\begin{tabular}{|l|r|r|r|r|}
\hline & \multicolumn{2}{|c|}{ Espagne } & \multicolumn{2}{c|}{ Italie } \\
\hline & Effectif & \% & \multicolumn{1}{c|}{ Effectif } & \multicolumn{1}{c|}{$\%$} \\
\hline Afrique subsaharienne & 150414 & 3,9 & 211283 & 7,9 \\
\hline Afrique du Nord & 535905 & 13,8 & 483705 & 18,1 \\
\hline Europe & 1593675 & 41,0 & 1261964 & 47,3 \\
\hline Amérique du Nord & 41887 & 1,1 & 16779 & 0,6 \\
\hline Amérique latine & 1367989 & 35,2 & 238882 & 8,9 \\
\hline Asie & 192323 & 5,0 & 454793 & 17,0 \\
\hline Océanie & 2380 & 0,1 & 2486 & 0,1 \\
\hline Apatride & nd & & 622 & 0,0 \\
\hline Total & 3884573 & 100,0 & 2670514 & 100,0 \\
\hline
\end{tabular}

Sources : www.ine.es (Espagne; Padron 2006).

www.istat.it (Italie ; Étrangers résidents déclarés 2006). 
Mais que représentent statistiquement les migrants venus du sud du Sahara dans ces nouveaux pays de destination, situés aux portes de l'Afrique ? La réponse est simple : une petite minorité. En 2006, en Italie, les personnes nées en Afrique subsaharienne représentaient environ $8 \%$ de la population immigrée ; elles étaient devancées de très loin par les ressortissants de pays d'Europe (47\%), d'Afrique du Nord (18\%) et d'Asie (17\%). La même année, en Espagne, la proportion des Subsahariens était de moins de $4 \%$, les Latino-américains $(35 \%)$ représentant le principal groupe d'immigration après les Européens (41\%). Encore faut-il souligner que ces données ne sont pas ou peu faussées par le statut légal des migrants. En Espagne, les migrants irréguliers sont en effet enregistrés dans les registres municipaux (Padron $)^{16}$. Quant aux données italiennes de 2006, même si elles ne peuvent prétendre à une stricte exhaustivité, elles intègrent les personnes régularisées en 1998 et 2002. Leur exactitude, concernant la répartition par origine des immigrés, est d'ailleurs confortée par les résultats d'une source alternative. Les Subsahariens représentaient en 2005 environ $9 \%$ de l'ensemble des étrangers, tous statuts de résidence — régulier ou irrégulier - confondus (Tableau 10) ${ }^{17}$.

Tableau 10 : Répartition de la population étrangère en Italie selon le statut de résidence (2005)

\begin{tabular}{|c|c|c|c|c|c|}
\hline & \multicolumn{2}{|c|}{$\begin{array}{l}\text { Étrangers (tous statuts } \\
\text { de résidence confondus) }\end{array}$} & \multicolumn{2}{|c|}{$\begin{array}{l}\text { Étrangers en situation } \\
\text { d'irrégularité }\end{array}$} & $\begin{array}{c}\text { Taux d'irrégularité } \\
(\%)\end{array}$ \\
\hline & Effectif (1) & $\%$ & Effectif (2) & $\%$ & $(2) /(1)$ \\
\hline Afrique subsaharienne & 286700 & 9 & 47200 & 9 & 16 \\
\hline Afrique du Nord & 625500 & 19 & 90500 & 17 & 14 \\
\hline Europe de l'Est & 1544500 & 46 & 286000 & 53 & 19 \\
\hline Amérique Latine & 321200 & 10 & 50000 & 9 & 16 \\
\hline Asie et Océanie & 579500 & 17 & 66700 & 12 & 12 \\
\hline Total & 3357400 & 100 & 540400 & 100 & 16 \\
\hline
\end{tabular}

Source : Blangiardo Gian Carlo, Farina Patrizia, 2007, Il Mezzogiorno dopo la grande regolarizzazione, Vol 3: Immagini e problematiche dell'immigrazione, Roma, Ministero della solidarieta sociale, p. 30-31.

http://www.solidarietasociale.gov.it/SolidarietaSociale/tematiche/Immigrazione/pubblicazioni

16 Il est dans l'intérêt des migrants irréguliers de s'enregistrer dans ces registres : d'une part, dans l'immédiat, c'est ce qui leur donne accès aux services publics ; d'autre part, lors des régularisations, c'est cet enregistrement qui témoigne de leur ancienneté de séjour en Espagne.

17 Les estimations de cette enquête sont basées sur la méthode dite du partage des poids, renommée en "échantillonnage de centres » dans le contexte de cette enquête. La méthode est décrite dans Groenewold (2004) et dans Mecatti (2004). 
Tableau 11 : Origine nationale des bénéficiaires des opérations de régularisation conduites dans les pays d'Europe méridionale (1997-2003)

\begin{tabular}{|l|c|c|c|c|c|c|}
\hline Régions d'origine & $\begin{array}{c}\text { Grèce } \\
(\mathbf{1 9 9 7 - 9 8})\end{array}$ & $\begin{array}{c}\text { Italie } \\
(\mathbf{1 9 9 8})\end{array}$ & $\begin{array}{c}\text { Italie } \\
(\mathbf{2 0 0 2})\end{array}$ & $\begin{array}{c}\text { Portugal } \\
(\mathbf{2 0 0 1 - 0 3})\end{array}$ & $\begin{array}{c}\text { Espagne } \\
(\mathbf{2 0 0 0})\end{array}$ & $\begin{array}{c}\text { Espagne } \\
(\mathbf{2 0 0 1})\end{array}$ \\
\hline Afrique subsaharienne & 0,9 & 13,9 & 4,9 & 15,1 & 13,7 & 6,2 \\
\hline Afrique du Nord & 1,9 & 19,7 & 12,2 & 1,3 & 33,8 & 17,7 \\
\hline Europe de l'Est & 85,1 & 36 & 58,6 & 54,8 & 13,0 & 19,6 \\
\hline Asie & 12,1 & 22,6 & 13,8 & 7,7 & 13,8 & 8,4 \\
\hline Amérique Latine & 0,0 & 7,8 & 10,3 & 21,1 & 25,7 & 48,1 \\
\hline Total & 100,0 & 100,0 & 100,0 & 100,0 & 100,0 & 100,0 \\
\hline
\end{tabular}

Source : Srozza S. et Zucchetti E. (eds.), 2006. Il Mezzogiorno dopo la grande regolarizzazione. Vecchi e nuovi volti della presenza migratoria, Milano, FrancoAngeli, 204 p.

Ainsi, en Espagne comme en Italie, les migrants originaires des pays situés au sud du Sahara constituaient encore en 2006 un groupe peu nombreux et proportionnellement moins important qu'en France. Ce résultat, qui ne peut être imputé à une négligence comptable des personnes en situation irrégulière, est sans doute dû au caractère relativement récent de cette migration, en particulier en Espagne ${ }^{18}$. Mais dans quelle mesure la population subsaharienne alimente-t-elle le nombre de migrants irréguliers de ces pays ? En Italie, en 2005, les Subsahariens représentaient $9 \%$ des migrants dépourvus de tout titre de séjour et leur taux d'irrégularité était de $16 \%$, un taux similaire à la moyenne calculée pour l'ensemble des origines (cf. Tableau 10). Les bilans des opérations de régularisation donnent un éclairage complémentaire mais néanmoins convergent : en comparaison des migrants originaires d'Europe de l'est, d'Amérique Latine ou d'Afrique du Nord, les Subsahariens représentent toujours une minorité dans l'ensemble de la population régularisée. Ils ont bénéficié - au minimum — de $0,9 \%$ des régularisations accordées en Grèce et — au maximum — de $15,1 \%$ de celles effectuées au Portugal. Et, là où des régularisations successives ont eu lieu, la proportion des Subsahariens régularisés a accusé une forte baisse : elle est passée de 14 à $5 \%$ en Italie entre 1998 et 2002, et de 14 à $6 \%$ en Espagne entre 2000 et 2001 (cf. Tableau 3). La régularisation espagnole de 2005, largement décriée par les voisins européens et notamment français, confirme le fait que l'immigration irrégulière en Espagne concerne avant tout des ressortissants d'Amérique latine et infirme l'idée d'une régularisation massive de Subsahariens : le Sénégal, le Nigeria et le Mali n'apparaissaient respectivement qu'en 13,18 et $19^{\text {ème }}$ position du classement par nationalité des demandeurs. À eux trois, ils cumulent seulement 3,4\% des demandes déposées pour un total de 24000 demandes (Figure 6). En fin de compte, ces résultats mettent bien en perspective le cas français : d'un côté, la sur-représentation subsaharienne

18 Pour une analyse détaillée des taux de croissance de la population subsaharienne en Espagne, en Italie, et aussi dans d'autres pays européens, voir De Haas (2007). 
parmi les régularisés en France apparaît comme une exception qui reste à expliquer ${ }^{19}$; d'un autre côté, la baisse accusée en France entre 1997-1998 et 1999-2006 de la part relative des personnes originaires d'Afrique noire dans l'ensemble de la population régularisée est convergente avec les tendances espagnoles et italiennes.

Figure 6 : Répartition par nationalité des demandes de régularisation des travailleurs étrangers en Espagne (2005)

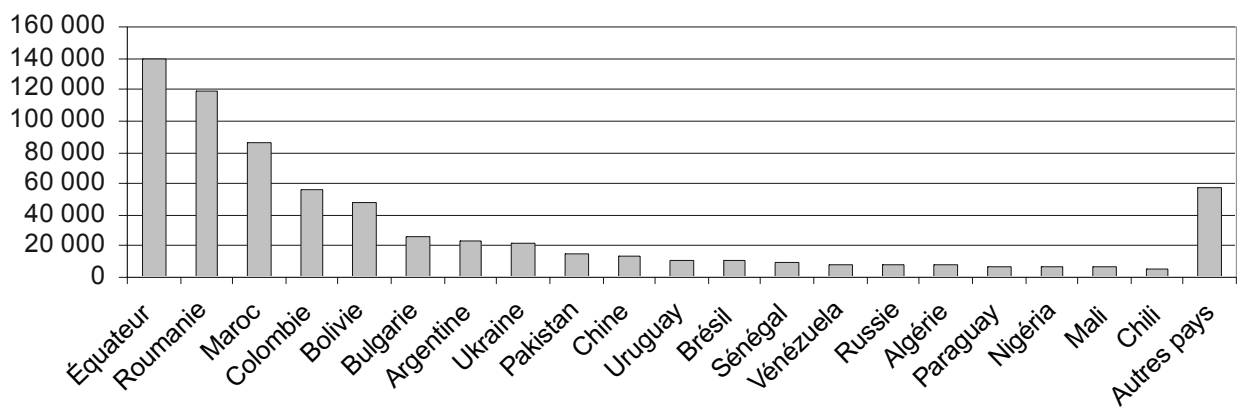

Source : Ministère du travail et des affaires sociales espagnol (2006) : «Balance del processo de Normalizacion Trabadojes extranjeros », $21 \mathrm{p}$.

\section{CONCLUSION}

Un fait dominant ressort systématiquement de ce tour d'horizon des statistiques sur les migrations internationales des Subsahariens: quelles que soient les mesures envisagées, les personnes originaires des pays d'Afrique noire forment toujours une minorité d'immigrants dans les pays développés, et en France en particulier. Du point de vue des zones de départ, même si la situation varie d'un pays à l'autre, l'émigration extra-continentale apparaît comme un phénomène démographique globalement marginal, les migrations entre pays voisins dominant largement les échanges internationaux. Ainsi, toutes les sources statistiques indiquent que la migration africaine, régulière et irrégulière, n'est massive ni du point de vue des pays de départ, ni du point de vue des pays d'arrivée. Il n’y a ni invasion, ni exode.

Ces regards statistiques portés sur les migrations internationales d'origine subsaharienne dévoilent ainsi le décalage entre l'ampleur des dispositifs politiques mis en place pour contrôler les flux et la réalité mesurée d'un phénomène finalement marginal. Pourtant, l'image d' « une Afrique exsangue et proche qui constitue un danger pour l'Europe redoutant le déferlement de hordes d'affamés et de demandeurs

19 De nouveau, on peut évoquer l'hypothèse selon laquelle cette surreprésentation est liée à des barrières administratives plus restrictives pour l'immigration des Subsahariens en France que dans les autres pays. Nous ne disposons malheureusement d'aucune donnée pour l'étayer. 
d'asile africain vers son territoire » demeure (Timera, 1997). Les résultats ici présentés fragilisent l'hypothèse souvent répandue selon laquelle la pauvreté de l'Afrique subsaharienne est la source d'un mouvement massif d'émigration subsaharienne vers les pays du Nord. Bien au contraire, il semblerait que la marginalisation du sous-continent dans le processus de mondialisation économique (échanges commerciaux et financiers) se reflète dans les mouvements de personnes.

Mais la visibilité artificiellement donnée aux migrations africaines par les discours politiques et médiatiques renforce la marginalisation de l'Afrique et de ses populations. Marginalisation diplomatique qui s'exprime dans les négociations interÉtats sur les migrations internationales par lesquelles les pays européens, en dépit de la faible importance numérique de flux subsahariens, imposent aux pays d'Afrique des accords et des techniques de gestion des flux qui sont souvent inappropriés ${ }^{20}$ et sans équivalent dans les autres régions du monde. Marginalisation sociale également, dans les pays de destination, où l'obsession politique des migrations subsahariennes apparaît comme un puissant facteur de stigmatisation d'une population déjà frappée par les stéréotypes racistes (Timera, 1997).

Mesurer les migrations n'est pas un exercice facile. Les divergences entre les sources statistiques signalées dans cet article en témoignent. Pour autant, même approximativement, il est possible de prendre la mesure des migrations africaines et de s'interroger. Justifient-elles le déploiement de moyens financiers extraordinaires pour garder les frontières méridionales de l'Europe ? Justifient-elles les pertes de vies humaines des candidats à la migration qui empruntent des chemins toujours plus dangereux pour contourner des barrières toujours plus hautes ? Justifient-elles de remettre en cause les processus de construction régionale engagés dans les pays d'origine ? Justifient-elles de nuire à la cohésion sociale dans les pays d'accueil ? Autant de questions qui devraient être intégrées à la réflexion dans l'évaluation des politiques publiques en matière d'immigration.

20 Par exemple, le contrôle des côtes sénégalaises par l'Agence Frontex, qui vise à interpeller les supposés migrants au moment de leur départ, est contraire à l'application de la Déclaration universelle des droits selon laquelle tout individu est libre de quitter le pays où il habite. Ce dispositif n'est d'ailleurs conforme à aucune disposition légale sénégalaise. Autre exemple : les pays européens, incitant les pays d'Afrique de l'ouest à contrôler davantage les sorties de leur territoire, les invitent également à contrôler davantage les entrées. Ils fragilisent ainsi les accords de libre circulation conclus au sein de la CEDEAO. La libre circulation à l'intérieur de l'Europe, ayant pour corollaire le renforcement des marges de l'Europe, conduit ainsi à restreindre la libre circulation à l'intérieur de l'Afrique. 


\section{Références bibliographiques}

ARDITTIS Solon, LACCZKO Frank (2008) Assessing the costs and impacts of migration policy: an international comparison. Genève, International organization for migration, $204 \mathrm{p}$.

AUDEBERT Cédric, ROBIN Nelly (2008) L'externalisation des frontières des « Nords » dans les eaux des «Suds » : l'exemple des dispositifs frontaliers américains et européens visant au contrôle de l'émigration caribéenne et subsaharienne, Poitiers, Migrinter-IRD, $12 \mathrm{p}$.

BLANGIARDO Gian Carlo, FARINA Patrizia (2007) Il Mezzogiorno dopo la grande regolarizzazione, in Vol 3 : Immagini e problematiche dell'immigrazione, Roma, Ministero della solidarieta sociale, $236 \mathrm{p}$.

BOCQUIER Philippe (1998) L'immigration ouest-africaine en Europe : une dimension politique sans rapport avec son importance démographique, La chronique du CEPED, juilletSeptembre $1998, \mathrm{n}^{\circ} 30$.

BORREL Catherine (2006) Enquêtes annuelles de recensement 2004 et 2005, INSEE Première $\mathrm{n}^{\circ}$ 1098, Août 2006.

CAMBRÉZY Luc (2001) Réfugiés et exilés : crise des sociétés, crise des territoires, Paris, Éd. des Archives contemporaines, 216 p.

CARLING Jorgen (2007) Migration Control and Migrant Fatalities at the Spanish-African Borders, International Migration Review, 41(2), pp. 316-343.

CASTLES Stephen (2004) The Factors that Make and Unmake Migration Policies, International Migration Review, 38(3), pp. 852-884.

DE HASS Hein (2007) The Myth of Invasion: Irregular Migration from West Africa to the Maghreb and the European Union, IMI Research Report, 79 p.

DELAUNAY Daniel, TAPINOS Georges (1998) La mesure de l'immigration clandestine en Europe : rapport de synthèse, Eurostat : Document de travail, Population et conditions sociales.

DOCQUIER Frédéric, MARFOUK Abdeslam (2006) International migration by educational attainment (1990-2000) - Release 1.1, In C. Ozden and M. Schiff (eds.), International Migration, Remittances and Development, Palgrave Macmillan: New York.

DUMONT Jean-Christophe, LEMAITRE Georges (2005) Counting Immigrants and Expatriates in OECD Countries: a new perspective, Paris, OECD social, employment and migration working papers $25,41 \mathrm{p}$.

GARSON Jean-Pierre (1992) Migration and Interdependence: The Migration System between France and Africa, in Mary M. Kritz, Lin Lean Lim, Hania Zlotnik (éd.), International migration systems: a global approach, Oxford, Clarendon Press, pp. 80-93.

GROENEWOLD George, BILSBORROW Richard (2004) Design of Samples for International Migration Surveys: Methodological Considerations, Practical Constraints and Lessons Learned from a Multi-Country Study in Africa and Europe, Population Association of America 2004, Boston.

Haut Conseil à l'Intégration (2008) Rapport statistique 2006 de l'Observatoire Statistique de l'Immigration et de l'Intégration, $97 \mathrm{p}$. http://www.assfam.com/IMG/pdf/Rapport_statistique_2006.pdf

Haut Commissariat aux Réfugiés (2006) Réfugiés. Tendances mondiales en 2005. Panorama statistique des populations réfugiées, de demandeurs d'asile, de personnes déplacées à l'intérieur de leur pays, d'apatrides et autres personnes relevant des compétences du HCR, Rapport de la Division des Services Opérationnels, Genève, 115 p.

Haut Commissariat aux Réfugiés (2000) Les réfugiés dans le monde. 50 ans d'ans d'actions humanitaires, Annexes statistiques, pp. 301-325. 
HERAN François (2007) Le temps des immigrés. Essai sur le destin de la population française, La république des idées, Seuil, $109 \mathrm{p}$.

INSEE (2002) La population immigrée étrangère en France, CD ROM : recensement 1999.

LUCAS Robert (2006) Migration and Economic Development in Africa: A Review of Evidence, Journal of African Economies 15, pp. 337-395.

MARIE Claude-Valentin (1983) L'immigration clandestine et le travail clandestin des étrangers en France à travers la régularisation des «sans papiers » de 1981-1982. (Résultats d'une enquête sur échantillon). Rapport présenté à Monsieur Pierre Beregovoy, Ministre des Affaires sociales et de la solidarité nationale.

MASSEY Douglas, DURAND Jorge et al. (2002) Beyond Smoke and Mirrors: Mexican Immigration in the Area of Economic Integration, New York, Russell Sage Foundation.

MECATTI Fulvia (2004) Échantillonnage de centres : stratégie d'enquête auprès des populations difficiles à échantillonner, Recueil du Symposium 2004 de Statistique Canada, Méthodes innovatrices pour enquêter des populations difficiles à joindre, $11 \mathrm{p}$.

Ministerio de trabajo y asuntos sociales (2006) Proceso de normalisacion de trabadojes extranjeros, Madrid, 2006, $21 \mathrm{p}$.

OCDE (2005) Tendances des migrations internationales et des politiques migratoires, SOPEMI, Edition 2004.

Office Français de Protection des Réfugiés et Apatrides (2007) Rapport annuel 2007, OFPRA, République Française, 74 p.

[Rapports 2001 à 2007 à télécharger :

http://www.ofpra.gouv.fr/index.html?xml_id=269\&dtd_id=10]

Office des Migrations Internationales (1994-2004) OMISTATS : Annuaire des migrations internationales, OMI-Paris.

PRIOUX France (2003) L'évolution démographique récente en France, Population, 4-5.

REGNARD Corinne (2006) Immigration et présence étrangère en France en 2005, Rapport annuel de la Direction de la Population et des Migrations, Ministère de l'Emploi, de la Cohésion sociale et du Logement, République Française, 260 p.

ROBIN Nelly (1996) Atlas des migrations ouest-africaines vers l'Europe, 1985-1993, Paris, Orstom Editions, $110 \mathrm{p}$.

SCHOORL Jeannette, HEERING Liesbeth, ESVELDT I., GROENEWOLD George, VAN DER ERF Rob, et al. (2000) Push and Pull Factors of International Migration: A Comparative Report, Luxembourg, Eurostat, European communities.

Secrétariat Général du Comité Interministériel de Contrôle de l'Immigration (2007) Les orientations de la politique de l'immigration, Rapport au parlement, Décembre 2007 (Chapitre 6 : L'immigration Irrégulière), $228 \mathrm{p}$.

SPAAN Ernst, VAN MOPPES David (2006) African Exodus? Trends and Patterns of International Migration in Sub-Saharan Africa, Radbout University, Working papers Migration and Development series, Report $n^{\circ} 4,43 \mathrm{p}$.

STROZZA Salvatore et ZUCCHETTI Eugenio (2006) Il Mezzogiorno dopo la grande Regolarizzazione, Vecchi e nuovi volti della presenza migratoria, Milano, FrancoAngeli, $204 \mathrm{p}$.

THIERRY Xavier (2004) Évolution récente de l'immigration en France et éléments de comparaison avec le Royaume Uni, Population 59(5), pp. 725-764.

THIERRY Xavier (2001) Les entrées d'étrangers en France de 1994 à 1999, Population, 3.

THIERRY Xavier (2000) Les entrées d'étrangers en France : évolutions statistiques et bilan de l'opération de régularisation exceptionnelle de 1997, Population, 3.

TIMERA Mahamet (1997) L'immigration africaine en France : regards des autres et repli sur soi, Politique africaine, $\mathrm{n}^{\circ}$ 67, $65 \mathrm{p}$. 


\section{ANNEXES \\ Annexe 1 : Vers une ré-estimation du nombre de Subsahariens en France en 1999 et des taux de croissance des périodes 1990-1999 et 1999-2004}

De l'aveu de l'INSEE, le recensement de 1999 aurait sous-estimé la population immigrée résidant en France (Borrel, 2006). En combinant les données des enquêtes annuelles de recensement publiées pour l'année 2004, avec celles d'AGDREF (période 1999-2004) et du REMUAO (période 1988-1992), il est possible de ré-estimer grossièrement la population africaine de 1999.

Que sait-on d'après ces sources ? (Tableau 1);

- En 2004, d'après le recensement, il y avait 570000 Subsahariens en France

- En 1990, d'après le REMUAO, il y aurait eu entre l'Afrique de l'ouest et l'Europe des 12 une sortie pour trois entrées ;

- Entre 1999 et 2004, d'après les données d'AGDREF traitées par Xavier Thierry, 170608 entrées de Subsahariens ont été enregistrées (Tableau 3).

Faisons l'hypothèse que le ratio sorties / entrées établi pour les flux entre l'Afrique de l'ouest et l'Europe en 1990 vaut approximativement pour les flux entre l'Afrique subsaharienne et la France entre 1999 et 2004. Appliquons arbitrairement un ratio plus restrictif que celui du REMUAO en comptant une sortie pour quatre entrées.

On peut alors estimer qu'il y a eu environ 40000 sorties de Subsahariens entre 1999 et 2004 (un quart des 170608 entrées comptabilisées grâce à AGDREF). Le solde migratoire de la période aurait été d'environ 130000 individus (170 608 entrées moins environ 40000 sorties). On peut donc estimer que la population subsaharienne en 1999 aurait été de 440000 individus (570 000 individus en 2004 auxquels on retranche 130 000), au lieu de 391854 . Les taux de croissance moyens seraient alors ramenées de 0 à 1,4\% par an entre 1990 et 1999 et de 7,7\% à $5,2 \%$ par an entre 1999 et 2004.

Par définition, les naissances n'entrent pas dans notre comptabilité : si un individu naît en France, il ne peut pas être immigré, quelque soit par ailleurs sa nationalité. On a cependant fait abstraction des décès des Subsahariens. Les comptabiliser conduirait à abaisser l'effectif de 1999 et à réduire davantage les écarts des taux de croissance entre les deux périodes intercensitaires.

Bien sûr, cet exercice comptable repose sur une hypothèse forte et n'a pas la valeur de données censitaires. Il offre néanmoins une estimation alternative du stock de la population subsaharienne en 1999 qui permet de relativiser les rythmes d'évolution de cette population entre 1990 et 2004. Notre estimation ne contredit pas la reprise de la croissance du stock de la population immigrée d'origine subsaharienne entre 1999 et 2004, mais elle la modère : elle serait plus proche de $5 \%$ que de $8 \%$. 


\section{Annexe 2 : Intégrer les migrants irréguliers subsahariens au total de la population résidant en France (1999)}

La disponibilité conjointe des données sur la vague de régularisation de 1997-1998 et du recensement de la population de 1999 offre une opportunité unique d'intégrer les migrants irréguliers dans une estimation de l'effectif de la population subsaharienne en France. Diverses hypothèses peuvent être formulées pour réaliser cette estimation. Celles que nous adoptons tendent systématiquement, pour l'année 1999, à majorer la part de la population africaine dans l'ensemble de la population immigrée $\left(\mathrm{X}_{\mathrm{AFR}}\right)$ que l'on estime de la façon suivante :

$$
X_{A F R}=\frac{P O P_{-} R E G_{A F R}+P O P_{-} R E F_{A F R}+P O P_{-} S D_{A F R}}{P O P_{-} R E G_{T O T}+P O P_{-} R E F_{T O T}+P O P_{-} S D_{T O T}}
$$

Où :

- POP_REG $G_{A F R}$ correspond à l'effectif de migrants réguliers d'origine subsaharienne en 1999. On prend ici non pas l'effectif du recensement (393 289), réputé sous-estimé, mais l'effectif ajusté à la hausse, conformément à l'annexe 1 (440 000).

- POP_REF $F_{A F R}$ est un premier terme de la population irrégulière : il correspond à l'effectif des personnes dont la demande de régularisation a été refusée. Tentons une estimation en raisonnant sur les résultats de l'opération de 1997-1998. D'après Thierry (2001), le taux de régularisation des Subsahariens était équivalent à celui de l'ensemble des migrants ayant déposé une demande. Sur 125000 demandes de régularisations déposées, toutes origines confondues, 90000 auraient été satisfaites selon les résultats ajustés par Xavier Thierry (2001). Le taux de refus peut donc être estimé à $28 \%$. D'après les données de l'ANAEM, 30572 Subsahariens ont été régularisés en 1997-1998 (tableau 4). On peut donc estimer que, en 1999, à l'issue de la vague de régularisation, l'effectif des individus non régularisés, suite à un refus, est d'environ 12000.

- POP_SD $D_{A F R}$ correspond au second terme de la population subsaharienne en situation irrégulière, à savoir l'ensemble des individus qui n'ont pas déposé une demande de régularisation. Aucune donnée ne nous permet de mesurer l'étendue de cette population. Faisons toutefois l'hypothèse très maximaliste qu'un Subsaharien irrégulier sur deux a pris le parti volontaire de rester «sans papier » à l'occasion de la vague de régularisation de 1997-1998. Il y aurait eu autant de demandeurs que de personnes n'ayant pas déposé une demande. L'effectif de $P O P \_S D_{A F R}$ serait donc d'environ 42600 personnes (30 572 régularisations ajoutées aux 12000 refus estimés ci-dessus).

- POP_REG $G_{T O T}$ correspond à l'effectif de l'ensemble de la population immigrée en 1999. Ne disposant pas de mesure alternative, nous reprenons l'effectif du recensement de la population (4 306 094, tableau 1). Notons que ne pas le réajuster à la hausse, contrairement à ce que nous avons fait pour $P O P_{-} R E G_{A F R}$, conduit à surestimer la part de la population d'origine subsaharienne $\left(\mathrm{X}_{\mathrm{AFR}}\right)$.

- POP_REF $F_{T O T}$ correspond à l'effectif total des immigrés dont la demande de régularisation n'a pas été satisfaite. D'après l'ANAEM, 76473 titres ont été délivrés en vertu de la régularisation de 1997-1998 (Tableau 4). En appliquant un taux de refus de 28 \%, on en déduit que le nombre total de déboutés a été de 29740 , toutes origines confondues.

- POP_SD $D_{T O T}$ correspond à la population immigrée irrégulière restée à l'écart de la vague de régularisation (individus n'ayant pas fait de demande de régularisation). Comme pour les Subsahariens $\left(P O P \_S D_{T O T}\right)$, cet effectif est inconnu. Là encore, adoptons l'hypothèse qui tend à maximiser la part des Subsahariens dans la population immigrée, en minimisant le dénominateur: faisons comme seuls des Subsahariens avaient fait le choix de ne pas solliciter une régularisation. Dans ce cas, $P O P_{-} S D_{T O T}=P O P_{-} S D_{A F R}$. 
Au total, notre estimation maximaliste de la population en situation irrégulière fait passer le nombre de Subsahariens résidant en France d'environ 440000 à un peu moins de 500000 individus. En définitive, migrants irréguliers inclus, la part de la population africaine dans l'ensemble de la population immigrée serait la suivante:

$$
X_{A F R}=\frac{440000+12000+42600}{4306094+29740+42600}=11,3 \%
$$

Ce résultat est peu éloigné de celui que l'on peut calculer simplement à partir des résultats du recensement $(9,1 \%$ d'après les chiffres du tableau 1$)$. Ainsi, les migrants venus du sud du Sahara demeurent une population largement minoritaire, y compris lorsque l'on inclut, de manière maximaliste, les individus dépourvus du droit de résidence. 


\title{
Ni invasion, ni exode. Regards statistiques sur les migrations d'Afrique subsaharienne
}

\author{
David LESSAULT et Cris BEAUCHEMIN
}

Les migrations africaines d'origine subsaharienne occupent aujourd'hui une place dominante dans les discours politiques et médiatiques portant sur l'immigration. Cette focalisation s'est traduite récemment par l'adoption d'une série de mesures politiques, policières et diplomatiques visant à réguler un flux considéré comme massif, voire invasif. Rassemblant des données statistiques éparses, cet article donne la mesure des migrations subsahariennes en adoptant à la fois les points de vue de l'immigration et de l'émigration, à l'échelle de la France mais aussi à l'échelle de l'Union européenne et de l'OCDE. L'état des connaissances statistiques est assorti d'une analyse des migrations irrégulières, généralement tenues pour responsables d'une sous-estimation officielle des flux. Les chiffres commentés sont sans équivoque : si les migrations subsahariennes sont extrêmement visibles dans les discours politiques et médiatiques, elles constituent pourtant un fait statistique minoritaire. Le paradoxe invite à s'interroger sur le bien fondé des politiques publiques en matière de gestion des migrations internationales.

\section{Neither Invasion nor Exodus. A statistical Overview of Migration from sub-Saharan Africa}

\section{David LESSAULT and Cris BEAUCHEMIN}

Migration from sub-Saharan Africa is today a key issue in the political debate on immigration and a focus of media attention. This concern was recently translated into action with the adoption of a series of political, law-enforcement and diplomatic measures to regulate inflows considered to have reached massive, even invasive, proportions. Bringing together previously dispersed statistical data, this article assesses the true extent of sub-Saharan migration, from the standpoints of both receiving and sending countries, on the scale of France, but also of the European Union and the OECD. The overview of statistical data is combined with an analysis of irregular migration, generally held responsible for official under-estimation of flows. The commented figures speak for themselves: although sub-Saharan migrants are extremely visible in the political and media discourse, they represent only a minority of the migrant population. This paradox challenges the cogency of public policy in the field of international migration control. 


\section{Ni invasión, ni éxodo. Estadisticos sobre las migraciones africanas de origen subsahariano}

\section{David LESSAULT y Cris BEAUCHEMIN}

Las migraciones africanas de origen subsahariano ocupan hoy un lugar dominante en los discursos políticos y medíaticos por lo que se refiere a la inmigración. Esta focalización se tradujo recientemente en la adopción de una serie de medidas políticas, policiales y diplomáticas destinadas a controlar un flujo considerado como macizo, o incluso invasivo. Reuniendo datos estadísticos dispersos, este artículo muestre la medida de las migraciones subsaharianas adoptando a la vez las opiniones de la inmigración y la emigración, a escala de Francia y también a escala de la Unión Europea y la OCDE. El estado de los conocimientos estadístico se combina con un análisis de las migraciones irregulares, generalmente consideradas como responsables de una infravaloración oficial de los flujos. Las cifras comentadas son inequívocas: si las migraciones subsaharianas son extremadamente visibles en los discursos políticos y medíaticos, constituyen con todo un hecho estadístico minoritario. La paradoja invita a preguntarse sobre el fundamento de las políticas públicas en cuanto a gestión de las migraciones internacionales. 